\title{
COMPARISON OF CONGRUENCES AND STRICT EQUIVALENCES FOR REAL, COMPLEX, AND QUATERNIONIC MATRIX PENCILS WITH SYMMETRIES*
}

\author{
LEIBA RODMAN ${ }^{\dagger}$
}

\begin{abstract}
The equivalence relations of strict equivalence and congruence of real and complex matrix pencils with symmetries are compared, depending on whether the congruence matrices are real, complex, or quaternionic. The obtained results are applied to comparison of congruences of matrices, over the reals, the complexes, and the quaternions.
\end{abstract}

Key words. Quaternionic matrices, Congruence, Strict equivalence.

AMS subject classifications. 15A33.

1. Introduction. Let $\mathbb{F}$ be the real field $\mathbb{R}$, the complex field $\mathbb{C}$, or the skew field of real quaternions $\mathbb{H}$. Fix an involutory antiautomorphism $\phi$ of $\mathbb{F}$, in other words, a bijective map $\phi: \mathbb{F} \longrightarrow \mathbb{F}$ having the properties that

$$
\phi(x y)=\phi(y) \phi(x) \quad \text { and } \quad \phi(x+y)=\phi(x)+\phi(y) \quad \forall \quad x, y \in \mathbb{F}
$$

and

$$
\phi(\phi(x))=x \quad \forall x \in \mathbb{F} .
$$

We assume furthermore that $\phi$ is continuous. (Note that in contrast to the complex case $\mathbb{F}=\mathbb{C}$, every antiautomorphism of $\mathbb{R}$ and of $\mathbb{H}$ is automatically continuous.) In particular, $\phi$ is the identity map if $\mathbb{F}=\mathbb{R}$, and $\phi$ is either the identity map or the complex conjugation if $\mathbb{F}=\mathbb{C}$. Denote by $\mathbb{F}^{m \times n}$ the set of all $m \times n$ matrices with entries in $\mathbb{F}$, and let $S^{\phi} \in \mathbb{F}^{n \times m}$ stand for the matrix obtained from $S \in \mathbb{F}^{m \times n}$ by applying entrywise the antiautomorphism $\phi$ to the transposed matrix $S^{T} \in \mathbb{F}^{n \times m}$.

We consider ordered pairs of matrices $(A, B)$, where $A, B \in \mathbb{F}^{m \times n}$, or equivalently, matrix pencils $A+t B$; here, $t$ is assumed to be a real valued independent variable, in particular, $t$ commutes with $A$ and $B$. The equivalence relation of strict equivalence is defined on the set of matrix pencils of fixed size $m \times n$ : Matrix pencils $A+t B$ and $A^{\prime}+t B^{\prime}$, where $A, B, A^{\prime}, B^{\prime} \in \mathbb{F}^{m \times n}$ are said to be strictly equivalent, or $\mathbb{F}$-strictly equivalent if $\mathbb{F}$ is to be emphasized, if there exist invertible matrices $S \in \mathbb{F}^{m \times m}$, $T \in \mathbb{F}^{n \times n}$ such that the equality

$$
S(A+t B) T=A^{\prime}+t B^{\prime}, \quad \forall t \in \mathbb{R},
$$

holds. Equality (1.1) clearly amounts to

$$
S A T=A^{\prime}, \quad S B T=B^{\prime} .
$$

\footnotetext{
* Received by the editors 21 April 2007. Accepted for publication 18 September 2007. Handling Editor: Roger A. Horn.

${ }^{\dagger}$ Department of Mathematics, College of William and Mary, Williamsburg, VA 23187-8795, USA (lxrodm@math.wm.edu). Research was supported in part by NSF grant DMS-0456625.
} 
For matrix pencils of square size $(m=n)$, also the equivalence relation of congruence (which depends on $\phi$ ) is defined: Matrix pencils $A+t B$ and $A^{\prime}+t B^{\prime}$, where $A, B, A^{\prime}, B^{\prime} \in \mathbb{F}^{n \times n}$ are said to be congruent, or more precisely $\phi$-congruent, if there exists an invertible matrix $S \in \mathbb{F}^{n \times n}$ such that the equality

$$
S^{\phi}(A+t B) S=A^{\prime}+t B^{\prime}, \quad \forall t \in \mathbb{R},
$$

holds. Of particular interest in this respect are the cases when $A$ and $B$ have certain symmetry properties with respect to $\phi$, namely $A= \pm A^{\phi}$ and $B= \pm B^{\phi}$ (the signs need not be the same for $A$ and $B$ ). It is easy to see that such symmetry properties are preserved under $\phi$-congruence.

Canonical forms of matrix pencils under strict equivalence, and canonical forms of matrix pencils with a symmetry property under congruence play a central role in many applications of matrix analysis. In the real and complex cases, these canonical forms are classical and can be found in numerous sources (see, e.g., [21] where many bibliographical references are provided, or more recent expositions [11], [12]). In the quaternionic case the literature on canonical forms is not nearly as extensive; we mention here the books [1], [2] (the latter contains a detailed treatment of the subject), and a list (far from compete) of relatively recent papers [4], [10], [16], [17], [18], [19], [20].

In the present paper we study comparisons between the equivalence relations of strict equivalence and congruence of matrix pencils with symmetry defined over different $\mathbb{F}$ 's. To formulate the problems precisely, let $\mathbb{F}^{\prime}$ be the real or complex field, with its own continuous involutory antiautomorphism $\phi^{\prime}$ (thus, $\phi^{\prime}$ is either the identity or (in the complex case) complex conjugation), and assume that $\mathbb{F}^{\prime}$ is a proper subfield of $\mathbb{F}$ and that $\phi$ when restricted to $\mathbb{F}^{\prime}$ coincides with $\phi^{\prime}$. The following are the main problems addressed in the present paper.

Problem 1.1. Assume that $A+t B$ and $A^{\prime}+t B^{\prime}$, where $A, B, A^{\prime}, B^{\prime} \in \mathbb{F}^{\prime n \times n}$, are matrix pencils with a symmetry property with respect to $\phi^{\prime}$.

(a) If $A+t B$ and $A^{\prime}+t B^{\prime}$ are $\phi$-congruent, in other words,

$$
S^{\phi}(A+t B) S=A^{\prime}+t B^{\prime}
$$

for some invertible matrix $S \in \mathbb{F}^{n \times n}$, what can we say about the $\phi^{\prime}$-congruence between $A+t B$ and $A^{\prime}+t B^{\prime}$ ?

(b) If $A+t B$ and $A^{\prime}+t B^{\prime}$ are $\mathbb{F}$-strictly equivalent, what can we say about the $\mathbb{F}^{\prime}$-strict equivalence of $A+t B$ and $A^{\prime}+t B^{\prime}$ ?

Problem 1.2. Under the hypotheses of Problem 1.1, identify those pencils $A+t B$ for which:

(a) $A$ pencil $A^{\prime}+t B^{\prime}$ is $\phi$-congruent to $A+t B$ if and only if $A^{\prime}+t B^{\prime}$ is $\phi^{\prime}$-congruent to $A+t B$.

(b) As in (a), but with respect to $\mathbb{F}$-strict equivalence and $\mathbb{F}^{\prime}$-strict equivalence.

Note that without the hypothesis on $A+t B$ and $A^{\prime}+t B^{\prime}$ having the symmetry property, the answers to parts 2 of Problems 1.1 and 1.2 are well known, and are given in terms of the Kronecker forms of the matrix pencils. 
As a corollary of a solution of Problem 1.2 we obtain necessary and sufficient conditions for a matrix $Y \in \mathbb{F}^{\prime n \times n}$ to have the following property: For any matrix $X \in \mathbb{F}^{\prime n \times n}$, if there exists an invertible $S \in \mathbb{F}^{n \times n}$ such that $S^{\phi} X S=Y$, then there exists also an invertible $R \in \mathbb{F}^{\prime n \times n}$ such that $R^{\phi^{\prime}} X R=Y$. See Theorems 3.2, 4.6, 7.6 for more details.

It will be convenient to introduce the classification of symmetries to be used in the present paper into 5 cases, as follows. Let $\sigma$ is a fixed involutory antiautomorphism, in short $i a a$, of $\mathbb{F}$ which is assumed to be continuous. The 5 cases are:

(I) $\mathbb{F}=\mathbb{R}, \sigma=$ id

(II) $\mathbb{F}=\mathbb{C}, \sigma=\mathrm{id}$

(III) $\mathbb{F}=\mathbb{C}, \sigma=$ complex conjugation;

(IV) $\mathbb{F}=\mathbb{H}, \sigma=$ quaternionic conjugation;

(V) $\mathbb{F}=\mathbb{H}, \sigma=$ iaa different from quaternionic conjugation.

In the sequel the iaa's of $\mathbb{H}$ different from the quaternionic conjugation will be termed nonstandard. We note that all nonstandard iaa's of $\mathbb{H}$ are similar to each other (and are not similar to the quaternionic conjugation): If $\tau_{1}, \tau_{2}$ are two such iaa's, then there exists an automorphism $\sigma$ of $\mathbb{H}$ such that

$$
\tau_{1}(\alpha)=\sigma^{-1}\left(\tau_{2}(\sigma(\alpha))\right), \quad \forall \alpha \in \mathbb{H} .
$$

This property, as well as and many other properties of iaa's to be used later on in the present paper, follows easily from the following known description of iaa's (see [15], [16], for example):

Proposition 1.3. A map $\phi: \mathbb{H} \longrightarrow \mathbb{H}$ is an iaa if and only if $\phi$ is real linear, and representing $\phi$ as a $4 \times 4$ real matrix with respect to the basis $\{1, \mathrm{i}, \mathrm{j}, \mathrm{k}\}$, we have:

$$
\phi=\left[\begin{array}{cc}
1 & 0 \\
0 & T
\end{array}\right]
$$

where either $T=-I_{3}$ (in which case $\phi$ is the quaternionic conjugation) or $T$ is a $3 \times 3$ real orthogonal symmetric matrix with eigenvalues $1,1,-1$.

In view of (1.3), indeed all nonstandard iaa's can be treated in one category (V).

We also note that if $\sigma$ is a nonstandard iaa of $\mathbb{H}$, then there is a unique (up to multiplication by -1 ) quaternion $\beta$ such that $\beta^{2}=-1$ (this equaity holds if and only if $\beta$ has norm 1 and zero real part) and $\sigma(\beta)=-\beta$. Conversely, for every $\beta \in \mathbb{H}$ with $\beta^{2}=-1$ there exists a unique nonstandard iaa $\sigma$ of $\mathbb{H}$ such that

$$
\sigma(\beta)=-\beta .
$$

We will often use the notation $(\mathrm{V})_{\beta}$ instead of $(\mathrm{V})$ to indicate that the iaa $\sigma$ in question is such that (1.4) holds. 
Let $A, B, A^{\prime}, B^{\prime} \in \mathbb{H}^{n \times n}$ (in particular, the case when $A, B, A^{\prime}, B^{\prime}$ are real or complex matrices is not excluded). We say that the matrix pencils $A+t B$ and $A^{\prime}+t B^{\prime}$ are (I)-, (II)-, (III)-, (IV)-, or $(\mathrm{V})_{\beta}$-congruent, respectively, if the equality

$$
S^{\sigma}(A+t B) S=A^{\prime}+t B^{\prime}
$$

holds for some invertible $S \in \mathbb{F}^{n \times n}$, where $\mathbb{F}$ and $\sigma$ are determined by (I), (II), (III), $(\mathrm{IV})$, or $(\mathrm{V})_{\beta}$, as the case may be, and where in addition in the case $(\mathrm{V})_{\beta}$ we assume that (1.4) holds. Clearly, each of these congruences is an equivalence relation.

We now review briefly the contents of the paper section by section. Section 2 is preliminary, and contains well known background information (to be used in the present paper) on quaternionic linear algebra. In Section 3 we study Problems 1.1 and 1.2 in the case when $\mathbb{F}^{\prime}$ is the real field. It turns out in particular, that for real matrix pencils with a symmetry, congruence (using the complex or quaternionic conjugation) with a complex or quaternionic congruence matrix is equivalent to that with a real congruence matrix, whereas $(\mathrm{V})$-congruence is the same as $\mathbb{R}$-strict equivalence. The main results there are Theorems 3.1 and 3.3. In Section 4 we study Problems 1.1 and 1.2 in the case when $\mathbb{F}^{\prime}=\mathbb{C}, \mathbb{F}=\mathbb{H}, \phi^{\prime}$ is the identity map, and $\phi$ is a nonstandard iaa. In this case, it turns out that $\mathbb{H}$-strict equivalence is the same as (IV)-congruence (Theorem 4.2). The comparison of congruences over the complex field and of congruences over the skew field of quaternions is given in terms of canonical forms of complex matrix pencils with symmetries (Theorem 4.4).

Next is the case when $\mathbb{F}^{\prime}=\mathbb{C}, \mathbb{F}=\mathbb{H}, \phi^{\prime}$ is the complex conjugation, and $\phi$ is the quaternionic conjugation, which is considered in Sections 5 and 6 . Here, it turns out that it is convenient to consider canonical forms for complex hermitian matrix pencils under congruence, depending on the particular symmetry of the given matrix pencil $A+t B$, where $A, B \in \mathbb{C}^{n \times n}$ : If $A=A^{*}, B=-B^{*}$, then we use the canonical form of the hermitian matrix pencil $A+t \mathrm{i} B$ ( $\mathrm{i}$ is the complex imaginary unit), and if $A=-A^{*}$, $B=-B^{*}$, then we use the canonical form of the hermitian matrix pencil $\mathrm{i} A+t \mathrm{i} B$. In the latter case, we obtain in particular the fact that $\mathbb{H}$-strict equivalence is the same as $\mathbb{C}$-strict equivalence (Theorem 5.3). The more difficult case when $A=A^{*}$, $B=-B^{*}$ is treated separately in Section 6 . Here the main results are Theorems 6.1 and 6.3 .

Finally, the situation when $\mathbb{F}^{\prime}=\mathbb{C}, \mathbb{F}=\mathbb{H}, \phi^{\prime}$ is the complex conjugation, and $\phi$ is a nonstandard iaa, is dealt with in Section 7. For skewhermitian complex matrix pencils, it turns out that $\mathbb{C}$-strict equivalence coincides with $\mathbb{H}$-strict equivalence, and an analogous statement holds for congruence (Theorem 7.3). The main result here is Theorem 7.4 which gives a complete characterization of congruences of hermitian matrix pencils $A+t \mathrm{i} B$ vs quaternionic congruences of pencils of the form $A+t B$.

We conclude the introduction with notation to be used throughout the paper. The standard imaginary units in $\mathbb{H}$ (the skew field of quaternions) will be denoted $\mathrm{i}, \mathrm{j}, \mathrm{k}$; thus, $\mathrm{i}^{2}=\mathrm{j}^{2}=\mathrm{k}^{2}=-1$ and $\mathrm{jk}=-\mathrm{kj}=\mathrm{i}, \mathrm{ij}=-\mathrm{ji}=\mathrm{k}, \mathrm{ki}=-\mathrm{ik}=\mathrm{j}$. For a quaternion $x=a_{0}+a_{1} \mathrm{i}+a_{2} \mathrm{j}+a_{3} \mathrm{k}, a_{0}, a_{1}, a_{2}, a_{3} \in \mathbb{R}$, we let $\mathfrak{R}(x):=a_{0}$ and 
$\mathfrak{V}(x):=a_{1} \mathrm{i}+a_{2} \mathrm{j}+a_{3} \mathrm{k}$ be the real and the vector parts of $x$, respectively. The conjugate quaternion $a_{0}-a_{1} \mathrm{i}-a_{2} \mathrm{j}-a_{3} \mathrm{k}$ is denoted by $\bar{x}$, and $|x|=\sqrt{a_{0}^{2}+a_{1}^{2}+a_{2}^{2}+a_{3}^{2}}$ stands for the norm of $x$. We denote by $\operatorname{diag}\left(X_{1}, X_{2}, \ldots, X_{p}\right)$, or by $X_{1} \oplus X_{2} \oplus \cdots \oplus X_{p}$, the block diagonal matrix with diagonal blocks $X_{1}, \ldots, X_{p}$ (in that order). For short, for a given $p \times q$ matrix $X$, we often use the notation

$$
X^{\oplus m}:=\operatorname{diag}(X, X, \ldots, X)=X \oplus X \oplus \cdots \oplus X,
$$

where $X$ appears $m$ times; thus, $X^{\oplus m}$ is a $m p \times m q$ matrix. The notation $A^{T}$, resp., $A^{*}$, stands for the transpose, resp., conjugate transpose, of the matrix $A$. We denote by $\operatorname{Span}_{\mathbb{R}}\{\alpha, \beta\}$ the real subspace of $\mathbb{H}$ spanned by $\alpha, \beta \in \mathbb{H}$.

The following matrices in standard forms and fixed notation that will be used. The subscript in notation for a square size matrix will always denote the size of the matrix.

$I$ and 0 (possibly with subscripts indicating the size) stand for the identity and the zero matrix, respectively.

The Jordan blocks:

$$
J_{m}(\lambda)=\left[\begin{array}{ccccc}
\lambda & 1 & 0 & \cdots & 0 \\
0 & \lambda & 1 & \cdots & 0 \\
\vdots & \vdots & \ddots & \ddots & 0 \\
\vdots & \vdots & & \lambda & 1 \\
0 & 0 & \cdots & 0 & \lambda
\end{array}\right] \in \mathbb{H}^{m \times m}, \quad \lambda \in \mathbb{H} .
$$

The real Jordan blocks:

$$
J_{2 m}(a \pm \mathrm{i} b)=\left[\begin{array}{ccccccc}
a & b & 1 & 0 & \cdots & 0 & 0 \\
-b & a & 0 & 1 & \cdots & 0 & 0 \\
0 & 0 & a & b & \cdots & 0 & 0 \\
0 & 0 & -b & a & \cdots & \vdots & \vdots \\
\vdots & \vdots & \vdots & \vdots & & 1 & 0 \\
\vdots & \vdots & \vdots & \vdots & & 0 & 1 \\
0 & 0 & 0 & 0 & \cdots & a & b \\
0 & 0 & 0 & 0 & \cdots & -b & a
\end{array}\right] \in \mathbb{R}^{2 m \times 2 m}, \quad a \in \mathbb{R}, \quad b \in \mathbb{R} \backslash\{0\} .
$$

Real symmetric matrices:

$$
F_{m}:=\left[\begin{array}{ccccc}
0 & \cdots & \cdots & 0 & 1 \\
\vdots & & & 1 & 0 \\
\vdots & & & & \vdots \\
0 & 1 & & & \vdots \\
1 & 0 & \cdots & \cdots & 0
\end{array}\right]=F_{m}^{-1}=F_{m}^{T},
$$




$$
G_{m}:=\left[\begin{array}{ccccc}
0 & \cdots & \cdots & 1 & 0 \\
\vdots & & & 0 & 0 \\
\vdots & & & & \vdots \\
1 & 0 & & & \vdots \\
0 & 0 & \cdots & \cdots & 0
\end{array}\right]=\left[\begin{array}{cc}
F_{m-1} & 0 \\
0 & 0
\end{array}\right]=G_{m}^{T} .
$$

Pencils of real $\varepsilon \times(\varepsilon+1)$ matrices:

$$
L_{\varepsilon \times(\varepsilon+1)}:=L_{\varepsilon \times(\varepsilon+1)}(t)=\left[\begin{array}{ccccc}
t & 1 & 0 & \cdots & 0 \\
0 & t & 1 & \cdots & 0 \\
\vdots & & \ddots & \ddots & \vdots \\
0 & 0 & \cdots & t & 1
\end{array}\right] .
$$

2. Preliminaries: Quaternionic linear algebra. In this section we recall basic facts about the quaternionic linear algebra, almost all without proofs. For more information and proofs, we refer the reader to [22], [23], [16], [5], [15], among many other sources. Recent interest in quaternionic linear algebra is motivated in part by applications in system and control [13], [14].

We start with similarity and congruence of quaternions. Quaternions $x, y \in \mathbb{H}$ are said to be similar, resp., congruent if $x=\alpha^{-1} y \alpha$, resp., $x=\bar{\alpha} y \alpha$ for some $\alpha \in \mathbb{H} \backslash\{0\}$.

Proposition 2.1. (1) Two quaternions $x$ and $y$ are similar if and only if $\mathfrak{R}(x)=$ $\mathfrak{R}(y)$ and $|\mathfrak{V}(x)|=|\mathfrak{V}(y)|$.

(2) Two quaternions $x$ and $y$ are congruent if and only if there exists $c>0$ such that $\mathfrak{R}(x)=c \mathfrak{R}(y)$ and $|\mathfrak{V}(x)|=c|\mathfrak{V}(y)|$. In particular, any two nonzero quaternions with zero real parts are congruent.

Part (1) here is well known, and part (2) follows easily from part (1) (see [18] for details.)

Next, consider the well known useful embeddings of $\mathbb{H}^{m \times n}$ into $\mathbb{R}^{4 m \times 4 n}$ and into $\mathbb{C}^{2 m \times 2 n}$, as follows. Write $x \in \mathbb{H}$ as a linear combination

$$
x=a_{0}+a_{1} \mathrm{i}+a_{2} \mathrm{j}+a_{3} \mathrm{k}, \quad a_{0}, a_{1}, a_{2}, a_{3} \in \mathbb{R} .
$$

Then we define

$$
\begin{gathered}
\mho_{\mathbb{R}}(x)=\left[\begin{array}{rrrr}
a_{0} & -a_{1} & a_{3} & -a_{2} \\
a_{1} & a_{0} & -a_{2} & -a_{3} \\
-a_{3} & a_{2} & a_{0} & -a_{1} \\
a_{2} & a_{3} & a_{1} & a_{0}
\end{array}\right] \in \mathbb{R}^{4 \times 4}, \\
\mho_{\mathbb{C}}(x)=\left[\begin{array}{cc}
a_{0}+a_{1} \mathrm{i} & a_{2}+a_{3} \mathrm{i} \\
-a_{2}+a_{3} \mathrm{i} & a_{0}-a_{1} \mathrm{i}
\end{array}\right] \in \mathbb{C}^{2 \times 2}
\end{gathered}
$$


and for

$$
X=\left[x_{\alpha, \beta}\right]_{\alpha=1, \beta=1}^{m, n} \in \mathbb{H}^{m \times n}
$$

define

$$
\mho_{\mathbb{R}}(X)=\left[\mho_{\mathbb{R}}\left(x_{\alpha, \beta}\right)\right]_{\alpha=1, \beta=1}^{m, n} \in \mathbb{R}^{4 m \times 4 n}, \quad \mho_{\mathbb{C}}(X)=\left[\mho_{\mathbb{C}}\left(x_{\alpha, \beta}\right)\right]_{\alpha=1, \beta=1}^{m, n} \in \mathbb{C}^{2 m \times 2 n} .
$$

The algebraic properties of the maps $\mho_{\mathbb{R}}$ and $\mho_{\mathbb{C}}$ are well known:

Proposition 2.2. The maps $\mho_{\mathbb{R}}$ and $\mho_{\mathbb{C}}$ are one-to-one ${ }^{*}$-homomorphisms of real algebras, i.e., denoting by $F$ either $\mathbb{R}$ or $\mathbb{C}$, we have:

$$
\begin{gathered}
\mho_{F}(a X+b Y)=a \mho_{F}(X)+b \mho_{F}(Y), \quad \forall X, Y \in \mathbb{H}^{m \times n}, \quad a, b \in \mathbb{R} ; \\
\mho_{F}(X Y)=\mho_{F}(X) \mho_{F}(Y), \quad \forall \quad X \in \mathbb{H}^{m \times n}, \quad Y \in \mathbb{H}^{n \times p} ; \\
\mho_{F}\left(X^{*}\right)=\left(\mho_{F}(X)\right)^{*}, \quad \forall X \in \mathbb{H}^{m \times n} .
\end{gathered}
$$

Note that the equality in $(2.1)$ takes the form $\mho_{F}\left(X^{*}\right)=\left(\mho_{F}(X)\right)^{T}$ if $F=\mathbb{R}$.

Next, consider pencils of quaternionic matrices $A+t B$, where $A$ and $B$ are $m \times n$ matrices with entries in $\mathbb{H}$, and $t$ is an independent real variable; in particular, $t$ commutes with the quaternionic matrices. Canonical form of the pencil $A+t B$ under strict equivalence:

$$
A+t B \quad \longrightarrow \quad P(A+t B) Q
$$

where $P \in \mathbb{H}^{m \times m}$ and $Q \in \mathbb{H}^{n \times n}$ are invertible matrices, is known as the Kronecker form, or $\mathbb{H}$-Kronecker form (if the skew field of quaternions is to be emphasized). Equivalently, it is the canonical form of ordered pairs of matrices $(A, B)$ under the group action $(A, B) \longrightarrow(P A Q, P B Q)$, with invertible quaternionic matrices $P$ and $Q$.

We describe the Kronecker form of quaternionic matrix pencils next.

TheOREM 2.3. Every pencil $A+t B$, where $A, B \in \mathbb{H}^{m \times n}$, is strictly equivalent to a matrix pencil in the block-diagonal form:

$$
\begin{aligned}
0_{u \times v} \oplus L_{\varepsilon_{1} \times\left(\varepsilon_{1}+1\right)} \oplus \cdots \oplus & L_{\varepsilon_{p} \times\left(\varepsilon_{p}+1\right)} \oplus L_{\eta_{1} \times\left(\eta_{1}+1\right)}^{T} \oplus L_{\eta_{q} \times\left(\eta_{q}+1\right)}^{T} \oplus \\
& \left(I_{k_{1}}+t J_{k_{1}}(0)\right) \oplus \cdots \oplus\left(I_{k_{r}}+t J_{k_{r}}(0)\right) \oplus \\
& \left(t I_{\ell_{1}}+J_{\ell_{1}}\left(\alpha_{1}\right)\right) \oplus \cdots \oplus\left(t I_{\ell_{s}}+J_{\ell_{s}}\left(\alpha_{s}\right)\right),
\end{aligned}
$$

where $\varepsilon_{1} \leq \ldots \leq \varepsilon_{p} ; \eta_{1} \leq \ldots \leq \eta_{q} ; k_{1} \leq \ldots \leq k_{r} ; \ell_{1} \leq \ldots \leq \ell_{s}$ are positive integers, and $\alpha_{1}, \ldots, \alpha_{s} \in \mathbb{H}$.

Moreover, the integers $u, v$, and $\varepsilon_{i}, \eta_{j}, k_{w}$ are uniquely determined by the pair $A, B$, and the part

$$
\left(t I_{\ell_{1}}+J_{\ell_{1}}\left(\alpha_{1}\right)\right) \oplus \cdots \oplus\left(t I_{\ell_{s}}+J_{\ell_{s}}\left(\alpha_{s}\right)\right)
$$


is uniquely determined by $A$ and $B$ up to a permutation of the diagonal blocks and up to replacing each $\alpha_{j}$ with any quaternion similar to $\alpha_{j}$.

The result of Theorem 2.3 is known, see [19] and [3], where it is stated in a less explicit form. A detailed proof of Theorem 2.3, following the standard approach for matrix pencils over fields as in [6] or [8], is given in [16].

We use the following terminology in connection with the Kronecker form (2.2) of the matrix pencil $A+t B$. The integers $\varepsilon_{1} \leq \ldots \leq \varepsilon_{p}$ and $\eta_{1} \leq \ldots \leq \eta_{q}$ are called the left indices and the right indices, respectively, of $A+t B$. The blocks $I_{k_{j}}+t J_{k_{j}}(0)$ are said to correspond to the eigenvalue at infinity, and the integers $k_{1} \leq \ldots \leq k_{r}$ are called the indices at infinity of $A+t B$. The quaternions $\alpha_{1}, \ldots, \alpha_{s}$ are called the eigenvalues of $A+t B$; they are uniquely determined up to permutation and similarity. Note that if $B$ is invertible, then the eigenvalues of $A+t B$ are exactly the eigenvalues of $B^{-1} A$ (or the eigenvalues of $A B^{-1}$ ). For a fixed eigenvalue $\alpha$ of $A+t B$, let $i_{1}<\ldots<i_{w}$ be all the subscripts in $(2.2)$ such that $\alpha_{i_{1}}, \ldots, \alpha_{i_{w}}$ are similar to $\alpha$; then the integers $\ell_{i_{1}}, \ldots, \ell_{i_{w}}$, with $\ell_{i_{j}}$ repeated as many times as there are blocks $J_{\ell_{i_{j}}}\left(\alpha_{i_{j}}\right)$ with $\alpha_{i_{j}}$ similar to $\alpha$ in (2.2), are called the indices of the eigenvalue $\alpha$ of $A+t B$.

Let $\alpha$ and $\alpha^{\prime}$ be eigenvalues of quaternionic matrix pencils $A+t B$ and $A^{\prime}+t B^{\prime}$, respectively, with corresponding indices $\ell_{i_{1}} \leq \cdots \leq \ell_{i_{w}}$ and $\ell_{i_{1}}^{\prime} \leq \cdots \leq \ell_{i_{w^{\prime}}}^{\prime}$, which are assumed (without loss of generality) to be arranged in the nondecreasing order. Then we say that the indices of $\alpha$ coincide with the indices of $\alpha^{\prime}$ (as the eigenvalues of $A+t B$ and $A^{\prime}+t B^{\prime}$, respectively) if $w^{\prime}=w$ and $\ell_{j}=\ell_{j}^{\prime}$ for $j=1,2, \ldots, w$. Similarly, the notion of coinciding indices at infinity of two quaternionic matrix pencils is introduced.

The connections between the Kronecker form of quaternionic pencils and the Kronecker form of their images under the maps $\mho_{\mathbb{R}}$ and $\mho_{\mathbb{C}}$ will be useful. By the real or complex Kronecker form, in short $\mathbb{R}$-Kronecker form or $\mathbb{C}$-Kronecker form, of a real or complex matrix pencil $X+t Y$ we mean the canonical form of $X+t Y$ under transformations

$$
X+t Y \quad \mapsto \quad S_{1}(X+t Y) S_{2},
$$

where $S_{1}$ and $S_{2}$ are invertible real or complex matrices, as the case may be (see [6] or [7, Chapter 2], for example).

THEOREM 2.4. Let (2.2) be the Kronecker form of a quaternionic pencil $A+t B$, and assume without loss of generality that $\alpha_{1}, \ldots, \alpha_{u}$ are real whereas

$$
\alpha_{u+1}=a_{u+1}+\mathrm{i} b_{u+1}, \ldots, \alpha_{s}=a_{s}+\mathrm{i} b_{s}, \quad a_{j}, b_{j} \in \mathbb{R}, \quad b_{j} \neq 0,
$$

are nonreal complex numbers, for $j=u+1, \ldots, s$. Then:

(a) The $\mathbb{R}$-Kronecker form of the real pencil $\mho_{\mathbb{R}}(A)+t \mho_{\mathbb{R}}(B)$ is given by

$$
\begin{aligned}
& 0_{4 u \times 4 v} \oplus \bigoplus_{j=1}^{p}\left(\left(L_{\varepsilon_{j} \times\left(\varepsilon_{j}+1\right)}\right)^{\oplus 4}\right) \oplus \bigoplus_{j=1}^{q}\left(\left(L_{\eta_{j} \times\left(\eta_{j}+1\right)}^{T}\right)^{\oplus 4}\right) \\
& \oplus \bigoplus_{j=1}^{r}\left[\left(I+t J_{k_{j}}(0)\right)^{\oplus 4}\right] \oplus \bigoplus_{j=1}^{u}\left[\left(t I+J_{m_{j}}\left(\alpha_{j}\right)\right)^{\oplus 4}\right] \bigoplus
\end{aligned}
$$




$$
\oplus \bigoplus_{j=u+1}^{s}\left[\left(t I+J_{2 m_{j}}\left(a_{j} \pm \mathrm{i} b_{j}\right)\right)^{\oplus 2}\right] .
$$

(b) The $\mathbb{C}$-Kronecker form of the complex pencil $\mho_{\mathbb{C}}(A)+t \mho_{\mathbb{C}}(B)$ is given by

$$
\begin{aligned}
& 0_{2 u \times 2 v} \oplus \bigoplus_{j=1}^{p}\left(\left(L_{\varepsilon_{j} \times\left(\varepsilon_{j}+1\right)}\right)^{\oplus 2}\right) \oplus \bigoplus_{j=1}^{q}\left(\left(L_{\eta_{j} \times\left(\eta_{j}+1\right)}^{T}\right)^{\oplus 2}\right) \\
& \oplus \bigoplus_{j=1}^{r}\left[\left(I+t J_{k_{j}}(0)\right)^{\oplus 2}\right] \oplus \bigoplus_{j=1}^{u}\left[\left(t I+J_{m_{j}}\left(\alpha_{j}\right)\right)^{\oplus 2}\right] \bigoplus \\
& \oplus \bigoplus_{j=u+1}^{s}\left[\begin{array}{cc}
t I+J_{m_{j}}\left(a_{j}+\mathrm{i} b_{j}\right) & 0 \\
0 & t I+J_{m_{j}}\left(a_{j}-\mathrm{i} b_{j}\right)
\end{array}\right] .
\end{aligned}
$$

This result can be easily obtained from [16, Theorem 3.8], which gives the Jordan forms of $\mho_{\mathbb{R}}(X)$ and $\mho_{\mathbb{C}}(X)$ in terms of the Jordan form of the quaternionic matrix $X$.

2.1. Comparison of strict equivalence. Let $\mathbb{F}$ be one of $\mathbb{R}, \mathbb{C}$, or $\mathbb{H}$. Two matrix pencils $A+t B$ and $A^{\prime}+t B^{\prime}$, where $A, B, A^{\prime}, B^{\prime} \in \mathbb{F}^{m \times n}$, are said to be $\mathbb{F}$ strictly equivalent if $A+t B=S\left(A^{\prime}+t B^{\prime}\right) T$ for some invertible matrices $S \in \mathbb{F}^{m \times m}$, $T \in \mathbb{F}^{n \times n}$.

PROPOSITION 2.5. Two real matrix pencils are $\mathbb{R}$-strictly equivalent if and only if they are $\mathbb{H}$-strictly equivalent, and hence if and only if they are $\mathbb{C}$-strictly equivalent.

Proof. Let $A, B, A^{\prime}, B^{\prime} \in \mathbb{R}^{m \times n}$, and assume that

$$
T(A+t B) S=A^{\prime}+t B^{\prime}
$$

for some invertible quaternionic matrices $T$ and $S$. Applying the map $\mho_{\mathbb{R}}$ to both sides of (2.3), and using Proposition 2.2, we see that $\mho_{\mathbb{R}}(A)+t \mho_{\mathbb{R}}(B)$ is $\mathbb{R}$-strictly equivalent to $\mho_{\mathbb{R}}\left(A^{\prime}\right)+t \mho_{\mathbb{R}}\left(B^{\prime}\right)$. It is easy to see that $\mho_{\mathbb{R}}(A)+t \mho_{\mathbb{R}}(B)$ is $\mathbb{R}$-strictly equivalent to $(A+t B)^{\oplus 4}$, in fact, the $\mathbb{R}$-strict equivalence matrices can be chosen to be suitable permutation matrices. Similarly, $\mho_{\mathbb{R}}\left(A^{\prime}\right)+t \mho_{\mathbb{R}}\left(B^{\prime}\right)$ is $\mathbb{R}$-strictly equivalent to $\left(A^{\prime}+t B^{\prime}\right)^{\oplus 4}$, and it remains to use a cancellation property (see [18]) to conclude that $A+t B$ is $\mathbb{R}$-strictly equivalent to $A^{\prime}+t B^{\prime}$. $\square$

Note that an analogue of Proposition 2.5 for the complex field does not hold: Two complex matrix pencils that are $\mathbb{H}$-strictly equivalent need not be $\mathbb{C}$-strictly equivalent, as scalar examples $t 1+\alpha, t 1+\bar{\alpha}$, where $\alpha \in \mathbb{C} \backslash \mathbb{R}$, show. Complex matrix pencils with this property can be easily identified, as shown in particular in the next proposition:

Proposition 2.6. Let there be given a complex matrix pencil $A+t B$, and let

$$
0_{u \times v} \oplus L_{\varepsilon_{1} \times\left(\varepsilon_{1}+1\right)} \oplus \cdots \oplus L_{\varepsilon_{p} \times\left(\varepsilon_{p}+1\right)} \oplus L_{\eta_{1} \times\left(\eta_{1}+1\right)}^{T} \oplus L_{\eta_{q} \times\left(\eta_{q}+1\right)}^{T} \oplus
$$


$\left(I_{k_{1}}+t J_{k_{1}}(0)\right) \oplus \cdots \oplus\left(I_{k_{r}}+t J_{k_{r}}(0)\right) \oplus\left(t I_{\ell_{1}}+J_{\ell_{1}}\left(\alpha_{1}\right)\right) \oplus \cdots \oplus\left(t I_{\ell_{s}}+J_{\ell_{s}}\left(\alpha_{s}\right)\right)$,

$$
\alpha_{j} \in \mathbb{C},
$$

be the complex Kronecker form for $A+t B$. Then, a complex matrix pencil $A^{\prime}+t B^{\prime}$ is $\mathbb{H}$-strictly equivalent to $A+t B$ if and only if the complex Kronecker form of $A^{\prime}+t B^{\prime}$ is obtained from (2.4) by replacing some (possibly none) of the blocks $J_{\ell_{j}}\left(\alpha_{j}\right)$ with non-real $\alpha_{j}$ by the blocks $J_{\ell_{j}}\left(\overline{\alpha_{j}}\right)$.

In particular, every complex matrix pencil which is $\mathbb{H}$-strictly equivalent to $A+t B$ is also $\mathbb{C}$-strictly equivalent to $A+t B$ if and only if all eigenvalues of $A+t B$ different from the infinity are real.

Proof. Without loss of generality we may assume that $A+t B$ is given by (2.4). The uniqueness part of Theorem 2.3 proves the "if" part of Proposition 2.6. For the part "only if", assume that

$$
A^{\prime}+t B^{\prime}=S(A+t B) T
$$

for some invertible quaternionic matrices $S$ and $T$. Then apply the map $\mho_{\mathbb{C}}$ to $(2.5)$ and use Theorem 2.4(b) to obtain the required property of the Kronecker form of $A^{\prime}+t B^{\prime}$ over $\mathbb{C}$.

3. Congruences of real symmetric or skewsymmetric matrix pairs. The main result on comparison of a congruence in the sense of one of (I), (II), (III), (IV), (V) versus another such congruence, of real matrix pairs with symmetries is given by the following theorem:

Theorem 3.1. Fix $\eta= \pm 1, \tau= \pm 1$. Let $A, B, A^{\prime}, B^{\prime} \in \mathbb{R}^{m \times m}$ be such that

$$
A^{T}=\eta A,\left(A^{\prime}\right)^{T}=\eta A^{\prime}, \quad B^{T}=\tau B,\left(B^{\prime}\right)^{T}=\tau B^{\prime} .
$$

Then, the statements (i), (ii), and (iii) below are equivalent. Also, the statements (iv), (v), (vi), and (vii) are equivalent.

(i) The matrix pencils $A+t B$ and $A^{\prime}+t B^{\prime}$ are (I)-congruent.

(ii) The matrix pencils $A+t B$ and $A^{\prime}+t B^{\prime}$ are (III)-congruent.

(iii) The matrix pencils $A+t B$ and $A^{\prime}+t B^{\prime}$ are (IV)-congruent.

(iv) The matrix pencils $A+t B$ and $A^{\prime}+t B^{\prime}$ are $\mathbb{R}$-strictly equivalent.

(v) The matrix pencils $A+t B$ and $A^{\prime}+t B^{\prime}$ are $\mathbb{H}$-strictly equivalent.

(vi) The matrix pencils $A+t B$ and $A^{\prime}+t B^{\prime}$ are (II)-congruent.

(vii) The matrix pencils $A+t B$ and $A^{\prime}+t B^{\prime}$ are $(\mathrm{V})_{\beta}$-congruent for some $\beta \in \mathbb{H}$, equivalently every $\beta \in \mathbb{H}$, such that $\mathfrak{R}(\beta)=0,|\mathfrak{V}(\beta)|=1$.

Moreover, if $\tau=\eta=-1$, then all statements (i) - (vii) are equivalent.

Proof. The implications (i) $\Longrightarrow$ (ii) $\Longrightarrow$ (iii) are obvious. We prove (iii) $\Longrightarrow$ (i). Let an invertible matrix $S \in \mathbb{H}^{m \times m}$ be such that equality

$$
S^{*}(A+t B) S=A^{\prime}+t B^{\prime}
$$

holds, and write $S=S_{0}+\mathrm{i} S_{1}+\mathrm{j} S_{2}+\mathrm{k} S_{3}$, where $S_{0}, S_{1}, S_{2}, S_{3}$ are real matrices. Then (3.2) takes the form

$$
U^{T}\left((A+t B)^{\oplus 4}\right) U=\left(A^{\prime}+t B^{\prime}\right)^{\oplus 4}
$$


where

$$
U:=\left[\begin{array}{cccc}
S_{0} & S_{1} & S_{2} & S_{3} \\
S_{1} & -S_{0} & S_{3} & -S_{2} \\
S_{2} & -S_{3} & -S_{0} & S_{1} \\
S_{3} & S_{2} & -S_{1} & -S_{0}
\end{array}\right] .
$$

Note that the matrix $U$ is invertible. Indeed, the equality

$$
U x=0, \quad x=\left[\begin{array}{llll}
x_{0} & x_{1} & x_{2} & x_{3}
\end{array}\right]^{T} \in \mathbb{R}^{4},
$$

is equivalent to $S\left(x_{0}-\mathrm{i} x_{1}-\mathrm{j} x_{2}-\mathrm{k} x_{3}\right)=0$, and therefore in view of the invertibility of $S, x$ must be the zero vector. Now apply a cancellation property [18] (which follows easily from the canonical form of real symmetric-skewsymmetric matrix pencils under (I)-congruence) to conclude that $A+t B$ and $A^{\prime}+t B^{\prime}$ are (I)-congruent.

Next, the implications (iv) $\Longrightarrow$ (v), (vii) $\Longrightarrow$ (v), and (vi) $\Longrightarrow$ (vii) are obvious (to see that (vi) $\Longrightarrow$ (vii), identify $\mathbb{C}$ with $\operatorname{Span}_{\mathbb{R}}\{1, q\} \subset \mathbb{H}$, where $q \in \mathbb{H}$ is such that $q^{2}=-1$ and $\sigma(q)=q$; existence of such $q$ is guaranteed for every nonstandard iaa $\sigma)$. Proposition 2.5 shows that in fact (iv) and (v) are equivalent. Thus, it remains to prove that (iv) $\Longrightarrow$ (vi). So, assume (iv) holds. As it follows from the canonical forms of pairs of real symmetric or skewsymmetric matrices under the (I)-congruence and $\mathbb{R}$-strict equivalence (see [11], [12], for example), there exist real invertible matrices $S$ and $T$ and pairs of matrices

$$
A_{j}, B_{j} \in \mathbb{R}^{n_{j} \times n_{j}}, \quad n_{1}+\cdots+n_{p}=m,
$$

such that

$$
S^{T}(A+t B) S=\oplus_{j=1}^{p} \delta_{j}\left(A_{j}+t B_{j}\right), \quad T^{T}\left(A^{\prime}+t B^{\prime}\right) T=\oplus_{j=1}^{p} \xi_{j}\left(A_{j}+t B_{j}\right),
$$

where for each $j$, the pair $\left(A_{j}, B_{j}\right)$ satisfies

$$
A_{j}^{T}=\eta A_{j}, \quad B_{j}^{T}=\tau B_{j},
$$

and $\delta_{1}, \ldots, \delta_{p}, \xi_{1}, \ldots, \xi_{p}$ are signs \pm 1 . It suffices to show that $\oplus_{j=1}^{p} \delta_{j}\left(A_{j}+t B_{j}\right)$ and $\oplus_{j=1}^{p} \xi_{j}\left(A_{j}+t B_{j}\right)$ are (II)-congruent. This is easy:

$$
\oplus_{j=1}^{p} \delta_{j}\left(A_{j}+t B_{j}\right)=\left(\oplus_{j=1}^{p} W_{j}\right)\left(\oplus_{j=1}^{p} \xi_{j}\left(A_{j}+t B_{j}\right)\right)\left(\oplus_{j=1}^{p} W_{j}\right),
$$

where $W_{j}=I_{n_{j}}$ if $\delta_{j}=\xi_{j}$ and $W_{j}=\mathrm{i} I_{n_{j}}$ if $\delta_{j} \neq \xi_{j}$.

Finally, the last statement follows from the fact (see, for example, [12, Theorem 5.1]) that if $\tau=\eta=-1$ then the real matrix pencils $A+t B$ and $A^{\prime}+t B^{\prime}$ satisfying (3.1) are $\mathbb{R}$-strictly equivalent if and only if they are (I)-congruent. $\square$

As an application of Theorem 3.1, we obtain a comparison result for congruence of real matrices over the reals vs congruence of real matrices over the quaternions:

THEOREM 3.2. If $X$ and $Y$ are real matrices such that $X=R^{*} Y R$ for some invertible $R \in \mathbb{H}^{n \times n}$, then also $X=S^{T} Y S$ for some invertible $S \in \mathbb{R}^{n \times n}$. 
For the proof apply the equivalence (i) $\Longleftrightarrow$ (iii) of Theorem 3.1 to the matrix pencils $\left(X+X^{T}\right) / 2+t\left(X-X^{T}\right) / 2$ and $\left(Y+Y^{T}\right) / 2+t\left(Y-Y^{T}\right) / 2$.

Next, leaving aside the case $\tau=\eta=-1$ which has been taken care of in Theorem 3.1 , we give necessary and sufficient conditions on the pencil $A+t B$ for all statements (i) - (vii) of Theorem 3.1 to be equivalent:

TheOREM 3.3. (a) Let there be given symmetric matrices $A, B \in \mathbb{R}^{m \times m}$. Then, the following statements are equivalent:

(i) for every pair $A^{\prime}, B^{\prime} \in \mathbb{R}^{m \times m}$ of symmetric matrices, the statements (i) (vii) of Theorem 3.1 are equivalent;

(ii) the $\mathbb{H}$-Kronecker form of $A+t B$ has no real eigenvalues and has no eigenvalues at infinity.

(iii) the $\mathbb{R}$-Kronecker form of $A+t B$ has no real eigenvalues and has no eigenvalues at infinity.

(b) Let there be given matrices $A, B \in \mathbb{R}^{m \times m}$, where $A$ is symmetric and $B$ is skewsymmetric. Then, the following statements are equivalent:

(iv) for every pair $A^{\prime}=A^{\prime T}, B^{\prime}=-B^{\prime T} \in \mathbb{R}^{m \times m}$, the statements (i) - (vii) of Theorem 3.1 are equivalent;

(v) the $\mathbb{H}$-Kronecker form of $A+t B$ has no nonzero eigenvalues with zero real parts, has no odd indices at infinity, and has no even indices corresponding to the eigenvalue zero (if zero is an eigenvalue);

(vi) the $\mathbb{R}$-Kronecker form of $A+t B$ has no nonzero pure imaginary eigenvalues, has no odd indices at infinity, and has no even indices corresponding to the eigenvalue zero (if zero is an eigenvalue).

Proof. By Proposition 2.5, (ii) and (iii) are equivalent, and (v) and (vi) are equivalent. The equivalence of (i) and (iii) follows from Theorem 3.1 and from the well known canonical forms of pairs of real symmetric matrices under (I)-congruence and $\mathbb{R}$-strict equivalence (see, e.g., [11, Theorem 9.1]). Indeed, the latter imply that (iii) holds precisely when for every pair $A^{\prime}=A^{\prime T}, B^{\prime}=B^{\prime T} \in \mathbb{R}^{m \times m}$, the matrix pencil $A^{\prime}+t B^{\prime}$ is $\mathbb{R}$-strictly equivalent to $A+t B$ if and only if $A^{\prime}+t B^{\prime}$ is (I)congruent to $A+t B$. Finally, the equivalence of (iv) and (vi) follows from Theorem 3.1 and [12, Corollary 12.3].

The result of Theorem 3.1 may be re-cast in terms of the canonical forms of matrix pencils under various strict equivalences and congruences. We will state explicitly only the result pertaining to the equivalence of (i) and (ii) in Theorem 3.1 (Corollary 3.5 below). First, for convenience of reference, we recall the well known canonical form for pairs of complex hermitian matrices under (III)-congruence (see, for example, [11], $[21])$.

PROPOSITION 3.4.

(a) Every matrix pencil $A+t B$, where

$$
A=A^{*} \in \mathbb{C}^{n \times n}, \quad B=B^{*} \in \mathbb{C}^{n \times n},
$$


is (III)-congruent to a complex hermitian matrix pencil of the form

$$
\begin{aligned}
& 0_{u \times u} \oplus\left(t\left[\begin{array}{ccc}
0 & 0 & F_{\varepsilon_{1}} \\
0 & 0 & 0 \\
F_{\varepsilon_{1}} & 0 & 0
\end{array}\right]+G_{2 \varepsilon_{1}+1}\right) \oplus \cdots \oplus\left(t\left[\begin{array}{ccc}
0 & 0 & F_{\varepsilon_{p}} \\
0 & 0 & 0 \\
F_{\varepsilon_{p}} & 0 & 0
\end{array}\right]+G_{2 \varepsilon_{p}+1}\right) \\
& \oplus \delta_{1}\left(F_{k_{1}}+t G_{k_{1}}\right) \oplus \cdots \oplus \delta_{r}\left(F_{k_{r}}+t G_{k_{r}}\right) \\
& \oplus \eta_{1}\left(\left(t+\alpha_{1}\right) F_{\ell_{1}}+G_{\ell_{1}}\right) \oplus \cdots \oplus \eta_{q}\left(\left(t+\alpha_{q}\right) F_{\ell_{q}}+G_{\ell_{q}}\right) \\
& \oplus\left(\left[\begin{array}{cc}
0 & \left(t+\beta_{1}\right) F_{m_{1}} \\
\left(t+\bar{\beta}_{1}\right) F_{m_{1}} & 0
\end{array}\right]+\left[\begin{array}{cc}
0 & G_{m_{1}} \\
G_{m_{1}} & 0
\end{array}\right]\right) \\
& \oplus \cdots \oplus\left(\left[\begin{array}{cc}
0 & \left(t+\beta_{s}\right) F_{m_{s}} \\
\left(t+\bar{\beta}_{s}\right) F_{m_{s}} & 0
\end{array}\right]+\left[\begin{array}{cc}
0 & G_{m_{s}} \\
G_{m_{s}} & 0
\end{array}\right]\right) \text {. }
\end{aligned}
$$

Here, $\varepsilon_{1} \leq \cdots \leq \varepsilon_{p}$ and $k_{1} \leq \cdots \leq k_{r}$ are positive integers, $\alpha_{j}$ are real numbers, $\beta_{j}$ are complex nonreal numbers with positive imaginary parts, $\delta_{1}, \ldots, \delta_{r}, \eta_{1}, \ldots, \eta_{q}$ are signs, each equal to +1 or -1 . blocks.

The form (3.3) is uniquely determined by $A+t B$ up to a permutation of constituent

(b) Every matrix pencil $A+t B$, where $A=A^{*} \in \mathbb{C}^{n \times n}, B=B^{*} \in \mathbb{C}^{n \times n}$, is $\mathbb{C}$-strictly equivalent to a unique (up to a permutation of constituent blocks) complex hermitian matrix pencil of the form (3.3), with all signs taken to be +1 .

The signs $\delta_{1}, \ldots, \delta_{r}, \eta_{1}, \ldots, \eta_{q}$ form the sign characteristic of the complex hermitian pencil $A+t B$. Thus, the sign characteristic associates a sign \pm 1 to every index of a real eigenvalue and of the eigenvalue at infinity of $A+t B$.

For complex matrix pencils $A+t B$, where $B=-B^{*}$ and $A= \pm A^{*}$, we will use in the sequel the canonical form as set forth in Proposition 3.4 for the hermitian matrix pencil $A+t(\mathrm{i} B)$ (if $A=A^{*}$ ) or i $A+t(\mathrm{i} B)$ (if $A=-A^{*}$ ). Note that complex matrix pencils $A+t B$ and $A^{\prime}+t B^{\prime}$, where $B=-B^{*}, B^{\prime}=-B^{\prime *}, A=A^{*}, A^{\prime}=A^{\prime *}$, are (III)-congruent if and only if the complex hermitian pencils $A+t(\mathrm{i} B)$ and $A^{\prime}+t(\mathrm{i} B)^{\prime}$ are (III)-congruent, i.e., have the same canonical form under Proposition 3.4. In the same vein, complex matrix pencils $A+t B$ and $A^{\prime}+t B^{\prime}$, where $B=-B^{*}, B^{\prime}=-B^{\prime *}$, $A=-A^{*}, A^{\prime}=-A^{\prime *}$, are (III)-congruent if and only if the complex hermitian pencils $(\mathrm{i} A)+t(\mathrm{i} B)$ and $\left(\mathrm{i} A^{\prime}\right)+t(\mathrm{i} B)^{\prime}$ have the same canonical form under Proposition 3.4. Thus, we obtain from the equivalence of (i) and (ii) of Theorem 3.1:

Corollary 3.5. Let $A, B, A^{\prime}, B^{\prime} \in \mathbb{R}^{m \times m}$ be such that

$$
A^{T}=\eta A,\left(A^{\prime}\right)^{T}=\eta A^{\prime}, \quad B^{T}=\tau B,\left(B^{\prime}\right)^{T}=\tau B^{\prime}, \quad \eta= \pm 1, \quad \tau= \pm 1 .
$$

Then the matrix pencils $A+t B$ and $A^{\prime}+t B^{\prime}$ are (I)-congruent if and only if the complex hermitian matrix pencils $\kappa(\eta) A+t(\kappa(\tau) B)$ and $\kappa(\eta) A^{\prime}+t\left(\kappa(\tau) B^{\prime}\right)$, where $\kappa(-1)=\mathrm{i}, \kappa(1)=1$, have the same canonical form under (III)-congruence.

4. Congruences of pairs of complex symmetric or skewsymmetric matrices. In this section we compare strict equivalences, (II)-congruences and $(\mathrm{V})_{\beta^{-}}$ congruences of pencils of symmetric or skewsymmetric complex matrices. Throughout this section we identify $\mathbb{C}$ with $\operatorname{Span}_{\mathbb{R}}\{1, \mathrm{i}\} \subset \mathbb{H}$, and we assume that $\beta \in \operatorname{Span}_{\mathbb{R}}\{\mathrm{j}, \mathrm{k}\}$. 
4.1. Main result: Comparison with quaternionic strict equivalence and congruence. From the assumptions set forth at the beginning of this section, we obviously have:

Proposition 4.1. If two complex matrix pencils $A+t B$ and $A^{\prime}+t B^{\prime}$ are (II)congruent, then they are also $(\mathrm{V})_{\beta}$-congruent.

We will be concerned in this section with the problem to what extent the converse statement holds, for pencils of complex symmetric or skewsymmetric matrices.

The key result of this section states that for complex pencils of symmetric or skewsymmetric matrices, $\mathbb{H}$-strict equivalence is the same as $(\mathrm{V})_{\beta^{-} \text {-congruence: }}$

Theorem 4.2. Fix $\eta= \pm 1, \tau= \pm 1$. Let $A, B, A^{\prime}, B^{\prime} \in \mathbb{C}^{m \times m}$ be such that $A^{T}=\eta A,\left(A^{\prime}\right)^{T}=\eta A^{\prime}, B^{T}=\tau B,\left(B^{\prime}\right)^{T}=\tau B^{\prime}$. Then the matrix pencils $A+t B$ and $A^{\prime}+t B^{\prime}$ are $\mathbb{H}$-strictly equivalent if and only if they are $(\mathrm{V})_{\beta}$-congruent.

The lengthy proof of Theorem 4.2 is relegated to the next subsection.

Comparison of (II)- and $(\mathrm{V})_{\beta}$-congruences will be given in terms of the well known canonical form for complex symmetric matrix pencils. The form is available in many sources, see [21], [9], for example; we follow here [21]:

Proposition 4.3. Every matrix pencil $A+t B$, where $A=A^{T} \in \mathbb{C}^{n \times n}, B=$ $B^{T} \in \mathbb{C}^{n \times n}$, is (II)-congruent to a complex symmetric matrix pencil of the form

$$
\begin{aligned}
& 0_{u \times u} \oplus\left(t\left[\begin{array}{ccc}
0 & 0 & F_{\varepsilon_{1}} \\
0 & 0 & 0 \\
F_{\varepsilon_{1}} & 0 & 0
\end{array}\right]+G_{2 \varepsilon_{1}+1}\right) \oplus \cdots \oplus\left(t\left[\begin{array}{ccc}
0 & 0 & F_{\varepsilon_{p}} \\
0 & 0 & 0 \\
F_{\varepsilon_{p}} & 0 & 0
\end{array}\right]+G_{2 \varepsilon_{p}+1}\right) \\
& \oplus\left(F_{k_{1}}+t G_{k_{1}}\right) \oplus \cdots \oplus\left(F_{k_{r}}+t G_{k_{r}}\right) \\
&4.1) \oplus\left(\left(t+\alpha_{1}\right) F_{\ell_{1}}+G_{\ell_{1}}\right) \oplus \cdots \oplus\left(\left(t+\alpha_{q}\right) F_{\ell_{q}}+G_{\ell_{q}}\right),
\end{aligned}
$$

where $\varepsilon_{1} \leq \cdots \leq \varepsilon_{p}$ and $k_{1} \leq \cdots \leq k_{r}$ are positive integers, and $\alpha_{j}$ are complex numbers. The form (4.1) is uniquely determined by $A+t B$ up to a permutation of constituent blocks.

A complete characterization of canonical forms of complex symmetric matrix pencils that are $(\mathrm{V})_{\beta}$-congruent to a fixed complex symmetric matrix pencil in a canonical form is given in the next theorem.

TheOREM 4.4. Let $A+t B$ be a complex symmetric matrix pencil, and let (4.1) be its canonical form under (II)-congruence. Then a complex symmetric matrix pencil $A^{\prime}+t B^{\prime}$ is $(\mathrm{V})_{\beta}$-congruent to $A+t B$ if and only if the canonical form of $A^{\prime}+t B^{\prime}$ under (II)-congruence is obtained from (4.1) by replacing some (or none) of the nonreal numbers $\alpha_{j}$ among $\alpha_{1}, \ldots, \alpha_{q}$ with their complex conjugates $\overline{\alpha_{j}}$.

For the proof observe that the canonical form of $A+t B$ under $(\mathrm{V})_{\beta}$-congruence is (4.1), up to permutation of blocks, and replacement of each $\alpha_{j}$ with its complex conjugate (see, for example, [16, Theorem 7.1]).

Results analogous to Theorem 4.4, with essentially the same proofs (using canonical forms of symmetric-skewsymmetric complex matrix pencils given in Proposition 4.7 below), hold also for pairs of complex skewsymmetric matrices, and for pairs of complex matrices, one of them being symmetric and the other skewsymmetric. We leave statements and proofs of these results to the interested readers. 
The following corollary is immediate from Theorem 4.4 and its analogues for pairs of complex skewsymmetric matrices and pairs of mixed complex symmetricskewsymmetric matrices:

Corollary 4.5. Fix $\eta= \pm 1, \tau= \pm 1$. A complex matrix pencil $A+t B$, where $A=\eta A^{T}, B=\tau B^{T}$, has the property that any complex symmetric pencil $A^{\prime}+t B^{\prime}$ with $A^{\prime}=\eta A^{\prime T}, B^{\prime}=\tau B^{\prime T}$ is (II)-congruent to $A+t B$ precisely when $A^{\prime}+t B^{\prime}$ is $(\mathrm{V})_{\beta}$-congruent to $A+t B$, if and only if $A+t B$ has no nonreal complex eigenvalues in its $\mathbb{C}$-Kronecker form.

In contrast to Theorem 3.2, the equivalence relation of congruence (using transposition) of complex matrices over $\mathbb{C}$ is generally different from the $\phi$-congruence of complex matrices over $\mathbb{H}$ (here, the iaa $\phi$ is such that $\phi(i)=i$ ). We characterize those complex matrices for which the two congruence relations turn out to be the same:

THEOREM 4.6. The following properties are equivalent for a complex matrix $Y \in \mathbb{C}^{n \times n}:$

(1) For some fixed nonstandard iaa $\phi$ such that $\phi(\mathrm{i})=\mathrm{i}$, if there exists an invertible quaternionic matrix $R \in \mathbb{H}^{n \times n}$ such that $X:=R^{\phi} Y R$ is complex, then $X=S^{T} Y S=S^{\phi} Y S$ for some invertible complex matrix $S$.

(2) If there exist (a) an invertible quaternionic matrix $R \in \mathbb{H}^{n \times n}$, and (b) a nonstandard iaa $\phi$ with $\phi(\mathrm{i})=\mathrm{i}$ such that $X:=R^{\phi} Y R$ is complex, then $X=S^{T} Y S=S^{\phi} Y S$ for some invertible complex matrix $S$.

(3) The matrix pencil $\left(Y+Y^{T}\right) / 2+t\left(Y-Y^{T}\right) / 2$ has no nonreal complex eigenvalues.

(4) The rank of the matrix $\left(Y+Y^{T}\right) / 2+z\left(Y-Y^{T}\right) / 2$ is the same for all $z \in \mathbb{C} \backslash \mathbb{R}$.

For the proof, apply Corollary 4.5 to the pencil $\left(Y+Y^{T}\right) / 2+t\left(Y-Y^{T}\right) / 2$, taking $\eta=1, \tau=-1$. The equivalence of (3) and (4) is obvious from the $\mathbb{C}$-Kronecker form of $\left(Y+Y^{T}\right) / 2+t\left(Y-Y^{T}\right) / 2$.

4.2. Proof of Theorem 4.2. We start with recalling the well known canonical forms for complex symmetric or skewsymmetric matrix pencils (for symmetric complex matrix pencils see Proposition 4.3). The expository paper [21] is the source for the next proposition.

PROPOSITION 4.7.

(a) Every matrix pencil $A+\lambda B$, where $A$ and $B$ are complex skew-symmetric matrices, is (II)-congruent to a pencil of skew-symmetric matrices of the form

$$
\begin{aligned}
0_{u \times u} & \oplus \bigoplus_{j=1}^{p}\left(t\left[\begin{array}{ccc}
0 & 0 & F_{\varepsilon_{j}} \\
0 & 0 & 0 \\
-F_{\varepsilon_{j}} & 0 & 0
\end{array}\right]+\left[\begin{array}{ccc}
0 & F_{\varepsilon_{j}} & 0 \\
-F_{\varepsilon_{j}} & 0 & 0 \\
0 & 0 & 0
\end{array}\right]\right) \\
& \oplus \bigoplus_{j=1}^{r}\left(\left[\begin{array}{cc}
0 & F_{k_{j}} \\
-F_{k_{j}} & 0
\end{array}\right]+t\left[\begin{array}{cc}
0 & G_{k_{j}} \\
-G_{k_{j}} & 0
\end{array}\right]\right) \oplus \\
& \oplus \bigoplus_{j=1}^{q}\left(\left(t+\alpha_{j}\right)\left[\begin{array}{cc}
0 & F_{\ell_{j}} \\
-F_{\ell_{j}} & 0
\end{array}\right]+\left[\begin{array}{cc}
0 & G_{\ell_{j}} \\
-G_{\ell_{j}} & 0
\end{array}\right]\right),
\end{aligned}
$$

where the positive integers $\varepsilon_{j}$ 's satisfy $\varepsilon_{1} \leq \cdots \leq \varepsilon_{p}$, and $\alpha_{j} \in \mathbb{C}$. The form (4.2) is uniquely determined by $A+t B$ up to permutations of diagonal blocks. 
(b) Every matrix pencil $A+t B$, where $A=A^{T}, B=-B^{T} \in \mathbb{C}^{n \times n}$, is (II)congruent to a direct sum of blocks of the following seven primitive types; several blocks of the same primitive type and/or the same size may be present in the direct sum:

$(\mathrm{sss} 0)$

a square size zero matrix.

$(\mathrm{sss} 1)$

$$
G_{2 \varepsilon+1}+t\left[\begin{array}{ccc}
0 & 0 & F_{\varepsilon} \\
0 & 0_{1} & 0 \\
-F_{\varepsilon} & 0 & 0
\end{array}\right]
$$

$(\operatorname{sss} 2)$

$$
F_{k}+t\left[\begin{array}{ccc}
0 & 0 & 0 \\
0 & 0 & F_{\frac{k-1}{2}} \\
0 & -F_{\frac{k-1}{2}} & 0
\end{array}\right], \quad k \text { odd. }
$$

$(\operatorname{sss} 3)$

$$
F_{k}+t\left[\begin{array}{cccc}
0_{1} & 0 & 0 & 0 \\
0 & 0 & 0 & F_{\frac{k-2}{2}} \\
0 & 0 & 0_{1} & 0 \\
0 & -F_{\frac{k-2}{2}} & 0 & 0
\end{array}\right], \quad k \text { even and } k / 2 \text { even. }
$$

$(\operatorname{sss} 4)$

$$
G_{\ell}+t\left[\begin{array}{cc}
0 & F_{\ell / 2} \\
-F_{\ell / 2} & 0
\end{array}\right], \quad \ell \text { even. }
$$

$(\mathrm{sss} 5)$

$$
\left[\begin{array}{cc}
0 & G_{\ell / 2} \\
G_{\ell / 2} & 0
\end{array}\right]+t\left[\begin{array}{cc}
0 & F_{\ell / 2} \\
-F_{\ell / 2} & 0
\end{array}\right], \quad \ell \text { even and } \ell / 2 \text { odd. }
$$

$(\operatorname{sss} 6)$

$$
\left[\begin{array}{cc}
0 & \alpha F_{\ell / 2}+G_{\ell / 2} \\
\alpha F_{\ell / 2}+G_{\ell / 2} & 0
\end{array}\right]+t\left[\begin{array}{cc}
0 & F_{\ell / 2} \\
-F_{\ell / 2} & 0
\end{array}\right]
$$

where $\ell$ even, $\alpha \in \mathbb{C} \backslash\{0\}$.

Moreover, the direct sum is uniquely determined by the pencil $A+t B$, up to permutation of the primitive blocks.

(d) Fix $\eta= \pm 1, \tau= \pm 1$. Let $A, B, A^{\prime}, B^{\prime} \in \mathbb{C}^{m \times m}$ be such that $A^{T}=\eta A$, $\left(A^{\prime}\right)^{T}=\eta A^{\prime}, B^{T}=\tau B,\left(B^{\prime}\right)^{T}=\tau B^{\prime}$. Then the matrix pencils $A+t B$ and $A^{\prime}+t B^{\prime}$ are $\mathbb{C}$-strictly equivalent if and only if the matrix pencils are (II)-congruent. 
Proof of Theorem 4.2. Clearly, we need to prove only the "only if" part. In the case $\tau=\eta=1$ this is clear in view of fact that symmetric quaternionic pencils are $\mathbb{H}$-strictly equivalent if and only if they are $(\mathrm{V})_{\beta}$-congruent $([16$, Theorem 7.1$])$.

Consider the case $\tau=\eta=-1$. Assume that $A+t B$ and $A^{\prime}+t B^{\prime}$ are $\mathbb{H}$ strictly equivalent. Since by Proposition 4.7 the relation of $\mathbb{C}$-strict equivalence of complex skew-symmetric pencils is the same as the relation of (II)-congruence, we may further replace $A+t B$ with its canonical form under (II)-congruence. In other words, we assume that $A+t B$ is given by (4.2). Note the following $(\mathrm{V})_{\beta}$-congruence relations (here $\sigma$ is a nonstandard iaa such that $\sigma(\beta)=-\beta$; recall that we assume $\left.\beta \in \operatorname{Span}_{\mathbb{R}}(\mathrm{j}, \mathrm{k})\right)$ :

$$
\begin{gathered}
S_{k_{j}}^{\sigma}\left[\begin{array}{cc}
0 & F_{k_{j}}+t G_{k_{j}} \\
-F_{k_{j}}-t G_{k_{j}} & 0
\end{array}\right] S_{k_{j}} \\
=\left(-\left(\beta F_{k_{j}}+t \beta G_{k_{j}}\right)\right) \oplus\left(\beta F_{k_{j}}+t \beta G_{k_{j}}\right) ; \\
S_{\ell_{j}}^{\sigma}\left(\left(t+\alpha_{j}\right)\left[\begin{array}{cc}
0 & F_{\ell_{j}} \\
-F_{\ell_{j}} & 0
\end{array}\right]+\left[\begin{array}{cc}
0 & G_{\ell_{j}} \\
-G_{\ell_{j}} & 0
\end{array}\right]\right) S_{\ell_{j}} \\
=\left(-\left(\left(t+\alpha_{j}\right) \beta F_{\ell_{j}}+\beta G_{\ell_{j}}\right)\right) \oplus\left(\left(t+\alpha_{j}\right) \beta F_{\ell_{j}}+\beta G_{\ell_{j}}\right), \quad \alpha_{j} \in \mathbb{R},
\end{gathered}
$$

where

$$
S_{m}=\frac{1}{\sqrt{2}}\left[\begin{array}{cc}
\beta I_{m} & -\beta I_{m} \\
I_{m} & I_{m}
\end{array}\right], \quad S_{m}^{\sigma}=\frac{1}{\sqrt{2}}\left[\begin{array}{cc}
-\beta I_{m} & I_{m} \\
\beta I_{m} & I_{m}
\end{array}\right]
$$

Comparing with the canonical form under $(\mathrm{V})_{\beta}$-congruence (see [16, Theorem 8.1]), we see that the canonical form of $A+t B$ under $(\mathrm{V})_{\beta}$-congruence is given by (4.2), where each block

$$
\left[\begin{array}{cc}
0 & F_{k_{j}} \\
-F_{k_{j}} & 0
\end{array}\right]+t\left[\begin{array}{cc}
0 & G_{k_{j}} \\
-G_{k_{j}} & 0
\end{array}\right]
$$

is replaced with the right hand side of (4.3), and each block

$$
\left(t+\alpha_{j}\right)\left[\begin{array}{cc}
0 & F_{\ell_{j}} \\
-F_{\ell_{j}} & 0
\end{array}\right]+\left[\begin{array}{cc}
0 & G_{\ell_{j}} \\
-G_{\ell_{j}} & 0
\end{array}\right]
$$

is replaced with the right hand side of (4.4). In other words, the blocks in the canonical form of $A+t B$ under $(\mathrm{V})_{\beta}$-congruence that correspond to the real eigenvalues and to the eigenvalue at infinity appear in pairs, and in each such pair the two blocks have opposite signs. Of course, the same property is valid also for the canonical form of $A^{\prime}+t B^{\prime}$ under $(\mathrm{V})_{\beta}$-congruence. Notice that $A+t B$ and $A^{\prime}+t B^{\prime}$ have the same canonical form under $\mathbb{H}$-strict equivalence, and that the canonical forms of quaternionic skewsymmetric (with respect to a nonstandard iaa) matrix pencils 
under $(\mathrm{V})_{\beta}$-congruence and under $\mathbb{H}$-strict equivalence can differ only in the signs associated with blocks corresponding to the real eigenvalues and to the eigenvalue at infinity (see [16, Theorem 8.1]). We obtain therefore that $A+t B$ and $A^{\prime}+t B^{\prime}$ have

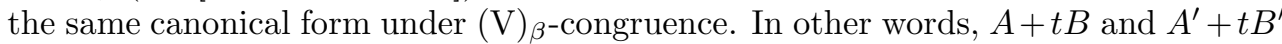
are $(\mathrm{V})_{\beta}$-congruent, as claimed.

Finally, consider the case $\eta=1, \tau=-1$ (the remaining case $\eta=-1, \tau=1$ can be easily reduced to the case under consideration by interchanging the roles of $A$ and $B)$. First of all, we will transform the primitive blocks (sss2) - (sss6) into different forms using $(\mathrm{V})_{\beta}$-congruence, so that the obtained forms are easily comparable to the canonical form of quaternionic symmetric-skewsymmetric pencils under $(\mathrm{V})_{\beta}$ congruence.

Claim 1. The block (sss5) is $(\mathrm{V})_{\beta}$-congruent to

$$
(G+t \beta F) \oplus(-(G+t \beta F)),
$$

where we let $G=G_{\ell / 2}, F=F_{\ell / 2}$, and recall that $\ell / 2$ is odd.

For the proof of the claim consider the matrix pencil

$$
\left[\begin{array}{cc}
0 & G \\
G & 0
\end{array}\right]+t\left[\begin{array}{cc}
0 & -\beta F \\
\beta F & 0
\end{array}\right]
$$

The matrix pencil (4.6) may be considered as a pencil of complex hermitian matrices, under the real linear map $\Psi$ of $\operatorname{Span}_{\mathbb{R}}\{1, \beta\}$ onto $\mathbb{C}$ via $1 \mapsto 1$ and $\beta \mapsto$ i. Transformations of the matrix pencil (4.6) of the form

$$
\left[\begin{array}{cc}
0 & G \\
G & 0
\end{array}\right]+t\left[\begin{array}{cc}
0 & -\beta F \\
\beta F & 0
\end{array}\right] \mapsto S^{\sigma}\left(\left[\begin{array}{cc}
0 & G \\
G & 0
\end{array}\right]+t\left[\begin{array}{cc}
0 & -\beta F \\
\beta F & 0
\end{array}\right]\right) S
$$

where $S$ is an invertible matrix with entries in $\operatorname{Span}_{\mathbb{R}}\{1, \beta\}$, amount to (III)- congruences under the map $\Psi$. We verify that the canonical form under (III)-congruence of (4.6), understood as a pencil of complex hermitian matrices, is equal to

$$
(G+t F) \oplus(-(G+t F)) .
$$

Indeed, since the $\mathbb{C}$-Kronecker form of (4.6) (again, under the map $\Psi)$ is $\left(t I+J_{\ell / 2}(0)\right) \oplus$ $\left(t I+J_{\ell / 2}(0)\right)$, it follows from Proposition 3.4 that the canonical form of (4.6) under (III)-congruence is

$$
\eta_{1}(G+t F) \oplus \eta_{2}(G+t F)
$$

where $\eta_{1}, \eta_{2}$ are signs \pm 1 . However, the case $\eta_{1} \eta_{2}=1$ is impossible, because if $\eta_{1} \eta_{2}=1$ holds then for large real values of $t$, the signature (= the difference between the number of positive eigenvalues, counted with multiplicities, and the number of negative eigenvalues, counted with multiplicities) of the hermitian matrix $\eta_{1}(G+$ $t F) \oplus \eta_{2}(G+t F)$ is not zero (this is where the hypothesis that $\ell / 2$ is odd is used), whereas for the hermitian matrix (4.6) the signature is equal to zero for all real $t$, a 
contradiction with the inertia theorem for hermitian matrices. Thus, the canonical form of (4.6) under (III)-congruence must be (4.7). In particular,

$$
S^{\sigma}\left[\begin{array}{cc}
0 & G \\
G & 0
\end{array}\right] S=\left[\begin{array}{cc}
G & 0 \\
0 & -G
\end{array}\right], \quad S^{\sigma}\left[\begin{array}{cc}
0 & -\beta F \\
\beta F & 0
\end{array}\right] S=\left[\begin{array}{cc}
F & 0 \\
0 & -F
\end{array}\right],
$$

for some invertible matrix $S$ with entries in $\operatorname{Span}_{\mathbb{R}}\{1, \beta\}$. Thus,

$$
S^{\sigma}\left[\begin{array}{cc}
0 & F \\
-F & 0
\end{array}\right] S=\left[\begin{array}{cc}
\beta F & 0 \\
0 & -\beta F
\end{array}\right],
$$

and the claim follows.

In a completely analogous way, the next claim is verified:

\section{Claim 2.}

(a) The block (sss2) is $(\mathrm{V})_{\beta}$-congruent to $F_{k}+t \beta G_{k}$; recall that $k$ is odd.

(b) The block (sss3) is $(\mathrm{V})_{\beta}$-congruent to

$$
\left(F_{k / 2}+t \beta G_{k / 2}\right) \oplus\left(-\left(F_{k / 2}+t \beta G_{k / 2}\right)\right) ;
$$

recall that $k / 2$ is even.

(c) The block (sss4) is $(\mathrm{V})_{\beta}$-congruent to $G_{\ell}+t \beta F_{\ell}$; recall that $\ell$ is even.

Our final claim concerns (sss6) with $\alpha \in \mathbb{C} \backslash\{0\}$ having zero real part:

Claim 3. The block

$$
\left[\begin{array}{cc}
0 & \alpha F_{q}+G_{q} \\
\alpha F_{q}+G_{q} & 0
\end{array}\right]+t\left[\begin{array}{cc}
0 & F_{q} \\
-F_{q} & 0
\end{array}\right]
$$

where $\alpha \in \mathbb{C} \backslash\{0\}$ has zero real part, is $(\mathrm{V})_{\beta}$-congruent to a block of the form

$$
\left(A_{0}+t B_{0}\right) \oplus\left(-\left(A_{0}+t B_{0}\right)\right),
$$

where $A_{0}=A_{0}^{\sigma} \in \mathbb{H}^{q \times q}$ and $B_{0}=-B_{0}^{\sigma} \in \mathbb{H}^{q \times q}$. Moreover, the $\mathbb{H}$-Kronecker form of the quaternionic pencil $A_{0}+t B_{0}$ consists of only one Jordan block of size $q \times q$ with eigenvalue $\alpha$ (or any similar eigenvalue).

Proof of Claim 3. First of all notice the equality (recall that $\beta \in \operatorname{Span}_{\mathbb{R}}\{\mathrm{j}, \mathrm{k}\}$, $\left.\alpha \in \operatorname{Span}_{\mathbb{R}}\{\mathrm{i}\}\right)$

$$
\alpha \beta=-\beta \alpha .
$$

Define the matrix

$$
Z=\operatorname{diag}\left(1,-1, \ldots,(-1)^{q-1}\right) .
$$

We obviously have $Z^{-1}=Z^{T}=Z$. In what follows, we denote $F=F_{q}, G=G_{q}$ for short.

Assume first that $q$ is odd. Then

$$
Z(F G) Z=-F G, \quad Z=F Z F, \quad Z G Z=-G .
$$


One now verifies that

$$
\begin{gathered}
{\left[\begin{array}{cc}
\beta I_{q} & -\beta Z \\
Z & I_{q}
\end{array}\right]^{\sigma}\left(\left[\begin{array}{cc}
0 & \alpha F_{q}+G_{q} \\
\alpha F_{q}+G_{q} & 0
\end{array}\right]+t\left[\begin{array}{cc}
0 & F_{q} \\
-F_{q} & 0
\end{array}\right]\right)\left[\begin{array}{cc}
\beta I_{q} & -\beta Z \\
Z & I_{q}
\end{array}\right]=} \\
2(\alpha \beta Z F+Z G \beta-(\beta Z F) t) \oplus 2(-\alpha \beta Z F+Z G \beta+(\beta Z F) t),
\end{gathered}
$$

and

$$
Z(-\alpha \beta Z F+Z G \beta+(\beta Z F) t) Z=-(\alpha \beta Z F+Z G \beta-(\beta Z F) t) .
$$

Assume now that $q$ is even. Then

$$
Z F Z=-F, \quad Z G Z=G,
$$

where $Z$ is defined by (4.10). Since $\sigma$ is a nonstandard iaa, it is easy to see that there exists $\gamma \in \mathbb{H}$ such that $\gamma^{2}=-1, \alpha \gamma=-\gamma \alpha$, and $\sigma(\gamma)=\gamma$. Now a straightforward verification shows that

$$
\begin{gathered}
{\left[\begin{array}{cc}
I_{q} & \gamma Z \\
\gamma Z & I_{q}
\end{array}\right]^{\sigma}\left(\left[\begin{array}{cc}
0 & \alpha F_{q}+G_{q} \\
\alpha F_{q}+G_{q} & 0
\end{array}\right]+t\left[\begin{array}{cc}
0 & F_{q} \\
-F_{q} & 0
\end{array}\right]\right)\left[\begin{array}{cc}
I_{q} & \gamma Z \\
\gamma Z & I_{q}
\end{array}\right]=} \\
(2(\gamma \alpha Z F+\gamma Z G-\gamma t Z F)) \oplus(2(\gamma \alpha Z F+\gamma Z G+\gamma t Z F)),
\end{gathered}
$$

and furthermore (note that $(\gamma Z)^{\sigma}=\gamma Z$ )

$$
(\gamma Z)(\gamma \alpha Z F+\gamma Z G+\gamma t Z F)(\gamma Z)=-(\gamma \alpha Z F+\gamma Z G-\gamma t Z F) .
$$

This completes verification of Claim 3.

Assume now that the complex pencils $A+t B$ and $A^{\prime}+t B^{\prime}$, where $A=A^{T}$, $B=-B^{T}, A^{\prime}=A^{\prime T} B^{\prime}=-B^{\prime T}$, are $\mathbb{H}$-strictly equivalent. Since by Proposition 4.7 the relation of $\mathbb{C}$-strict equivalence of complex symmetric-skewsymmetric pencils is the same as the relation of (II)-congruence, we may replace $A+t B$ with its canonical form under (II)-congruence, in other words, we may assume that $A+t B$ is a direct sum of primitive blocks of types (sss0) - (sss6). In view of Claims $1-3$, under the $(\mathrm{V})_{\beta}$-congruence, the blocks with eigenvalue zero having odd sizes, the blocks with eigenvalue at infinity having even sizes, and the blocks with nonzero complex eigenvalues having zero real parts, appear in pairs with opposite signs for each of the two blocks in every such pair. The same statement holds for $A^{\prime}+t B^{\prime}$ as well.

Now observe that the canonical form under $(\mathrm{V})_{\beta}$-congruence and the canonical form under $\mathbb{H}$-strict equivalence of a quaternionic matrix pencil

(4.11) $X+t Y, \quad X=X^{\sigma} \in \mathbb{H}^{m \times m}, \quad Y=-Y^{\sigma} \in \mathbb{H}^{m \times m}, \quad \sigma$ nonstandard iaa

may differ, apart from a permutation of blocks, only in signs \pm 1 that attached precisely to the blocks with eigenvalue zero having odd sizes, the blocks with eigenvalue at infinity having even sizes, and the blocks with nonzero eigenvalues having zero real parts. (See, for example, [4] or [17, Theorem 3.1] for canonical forms under $(\mathrm{V})_{\beta^{-}}$ congruence vs canonical forms under $\mathbb{H}$-strict equivalence for quaternionic matrix pencils with the symmetry property 4.11.) In view of the statement in the preceding paragraph, we are done. 
5. Congruences of pairs of complex hermitian or skewhermitian matrices I. In this and the next section we deal with comparison of (III)-congruence and congruences over the quaternions for matrix pencils $A+t B$, where $A= \pm A^{*}$ and $B= \pm B^{*}$ are complex matrices. In all cases, we use the canonical form for (III)-congruence of complex hermitian matrix pencils; if $A=A^{*}$ and $B=-B^{*}$, the canonical from for $A+t(\mathrm{i} B)$ will be used, and if $A=-A^{*}$ and $B=-B^{*}$, the canonical form for i $A+t(\mathrm{i} B)$ will be used. (We will not consider separately the case $A=-A^{*}$ and $B=B^{*}$ as it can be reduced to the case $A=A^{*}$ and $B=-B^{*}$ by interchanging the roles of $A$ and $B$.) For congruences over quaternions in this and the next section we use (IV)-congruence; in Section 7 the $(\mathrm{V})_{\mathrm{i}}$-congruence will be used.

An analogue of Theorem 3.1 (for $\eta=\tau=1$ ) holds in the complex case, with the transposes replaced by conjugate transposes:

Theorem 5.1. Let $A, B, A^{\prime}, B^{\prime} \in \mathbb{C}^{m \times m}$ be hermitian matrices. Then:

(a) The complex matrix pencils $A+t B$ and $A^{\prime}+t B^{\prime}$ are (IV)-congruent if and only if they are (III)-congruent.

(b) $A+t B$ and $A^{\prime}+t B^{\prime}$ are $\mathbb{H}$-strictly equivalent if and only if $A+t B$ and $A^{\prime}+t B^{\prime}$ are $\mathbb{C}$-strictly equivalent.

It will be convenient to prove a lemma first.

Lemma 5.2. Every complex hermitian matrix pencil $A_{0}+t B_{0}$ is (III)-congruent to its conjugate $\overline{A_{0}}+t \overline{B_{0}}$.

Proof. It is easy to see that we need to consider only the case when $A_{0}+t B_{0}$ is in the canonical form of complex hermitian matrix pencils under (III)-congruence, and then we may assume that $A_{0}+t B_{0}$ coincides with a primitive block. The primitive blocks for this canonical form are given in Proposition 3.4; they are either real, in which case the statement of the lemma is trivial, or they have the form $\left[\begin{array}{cc}0 & X \\ X & 0\end{array}\right]$, where $X$ is a square size complex matrix, in which case the congruence

$$
\left[\begin{array}{ll}
0 & I \\
I & 0
\end{array}\right]\left[\begin{array}{cc}
0 & X \\
X & 0
\end{array}\right]\left[\begin{array}{cc}
0 & I \\
I & 0
\end{array}\right]=\left[\begin{array}{cc}
0 & \bar{X} \\
X & 0
\end{array}\right]
$$

completes the proof.

Proof of Theorem 5.1. Part (a). Again, we only prove the "only if" part; thus, suppose that $A+t B$ and $A^{\prime}+t B^{\prime}$ are (IV)-congruent. Using the map $\mho_{\mathbb{C}}$ and Proposition 2.2, we see (applying the congruence with a suitable permutation matrix) that the complex hermitian $2 m \times 2 m$ matrix pencils

$$
(A+t B) \oplus(\bar{A}+t \bar{B}) \text { and } \quad\left(A^{\prime}+t B^{\prime}\right) \oplus\left(\overline{A^{\prime}}+t \overline{B^{\prime}}\right)
$$

are (III)-congruent. Using Lemma 5.2 , it follows that $(A+t B)^{\oplus 2}$ is (III)-congruent to $\left(A^{\prime}+t B^{\prime}\right)^{\oplus 2}$. Now apply a cancellation property (see [18]).

Part (b). Assume that $A+t B$ and $A^{\prime}+t B^{\prime}$ are $\mathbb{H}$-strictly equivalent. By Proposition 2.6, the $\mathbb{C}$-Kronecker form of $A+t B$ is obtained from the $\mathbb{C}$-Kronecker form of $A^{\prime}+t B^{\prime}$ by replacing some of Jordan blocks $J_{m}(\alpha)$ corresponding to non-real eigenvalues $\alpha$ with $J_{m}(\bar{\alpha})$.

On the other hand, it follows from Proposition 3.4 that for a complex matrix pencil of hermitian matrices, the $\mathbb{C}$-Kronecker form of the pencil is symmetric with 
respect to complex conjugation: If $\alpha$ is an eigenvalue, then so is also $\bar{\alpha}$, and the indices of $\alpha$ coincide with the indices of $\bar{\alpha}$. Combining this fact with the observation in the preceding paragraph, we conclude that $\mathbb{C}$-Kronecker forms of $A+t B$ and $A^{\prime}+t B^{\prime}$ must be the same.

If at least one of $\eta$ and $\tau$ is equal to -1 , then the situation with the complex analogues of Theorem 3.1 is more involved. We present here the case when $\eta=\tau=$ -1 , and relegate the remaining case when $\eta \tau=-1$ to a later section 6 .

TheOREM 5.3. (a) Let $A, A^{\prime}, B, B^{\prime} \in \mathbb{C}^{n \times n}$ be complex skew-hermitian matrices. Then, if the complex matrix pencils $A+t B$ and $A^{\prime}+t B^{\prime}$ are $\mathbb{H}$-strictly equivalent, and if (3.3) is the canonical form of the hermitian pencil $\mathrm{i} A+t(\mathrm{i} B)$ under (III)-congruence, then the canonical form of the hermitian pencil $\mathrm{i} A^{\prime}+t\left(\mathrm{i} B^{\prime}\right)$ under (III)-congruence is

$$
\begin{aligned}
& 0_{u \times u} \oplus\left(t\left[\begin{array}{ccc}
0 & 0 & F_{\varepsilon_{1}} \\
0 & 0 & 0 \\
F_{\varepsilon_{1}} & 0 & 0
\end{array}\right]+G_{2 \varepsilon_{1}+1}\right) \oplus \cdots \oplus\left(t\left[\begin{array}{ccc}
0 & 0 & F_{\varepsilon_{p}} \\
0 & 0 & 0 \\
F_{\varepsilon_{p}} & 0 & 0
\end{array}\right]+G_{2 \varepsilon_{p}+1}\right) \\
& \oplus \delta_{1}^{\prime}\left(F_{k_{1}}+t G_{k_{1}}\right) \oplus \cdots \oplus \delta_{r}^{\prime}\left(F_{k_{r}}+t G_{k_{r}}\right) \\
& \oplus \eta_{1}^{\prime}\left(\left(t+\alpha_{1}\right) F_{\ell_{1}}+G_{\ell_{1}}\right) \oplus \cdots \oplus \eta_{q}^{\prime}\left(\left(t+\alpha_{q}\right) F_{\ell_{q}}+G_{\ell_{q}}\right) \\
& \oplus\left(\left[\begin{array}{cc}
0 & \left(t+\beta_{1}\right) F_{m_{1}} \\
\left(t+\bar{\beta}_{1}\right) F_{m_{1}} & 0
\end{array}\right]+\left[\begin{array}{cc}
0 & G_{m_{1}} \\
G_{m_{1}} & 0
\end{array}\right]\right) \\
& \oplus \cdots \oplus\left(\left[\begin{array}{cc}
0 & \left(t+\beta_{s}\right) F_{m_{s}} \\
\left(t+\bar{\beta}_{s}\right) F_{m_{s}} & 0
\end{array}\right]+\left[\begin{array}{cc}
0 & G_{m_{s}} \\
G_{m_{s}} & 0
\end{array}\right]\right)
\end{aligned}
$$

for some choice of the signs

$$
\delta_{1}^{\prime}, \cdots, \delta_{r}^{\prime}, \eta_{1}^{\prime}, \ldots, \eta_{q}^{\prime} \in\{1,-1\}
$$

(b) The complex skew-hermitian pencils obtained from (5.1) by multiplying with -i are (IV)-congruent to each other, for any choice of the signs $\delta_{1}^{\prime}, \cdots, \delta_{r}^{\prime}, \eta_{1}^{\prime}, \ldots, \eta_{q}^{\prime}$.

(c) Let $A, A^{\prime}, B, B^{\prime} \in \mathbb{C}^{n \times n}$ be complex skew-hermitian matrices. Then the complex matrix pencils $A+t B$ and $A^{\prime}+t B^{\prime}$ are $\mathbb{H}$-strictly equivalent if and only if they are $\mathbb{C}$-strictly equivalent if and only if they are (IV)-congruent.

Proof. Part (a). The complex hermitian pencils $\mathrm{i} A+t(\mathrm{i} B)$ and $\mathrm{i} A^{\prime}+t\left(\mathrm{i} B^{\prime}\right)$ are clearly $\mathbb{H}$-strictly equivalent, therefore by Proposition 2.6 the $\mathbb{C}$-Kronecker forms of $\mathrm{i} A+t(\mathrm{i} B)$ and $\mathrm{i} A^{\prime}+t\left(\mathrm{i} B^{\prime}\right)$ are obtained from each other by replacing some Jordan blocks $J_{\ell_{j}}\left(\alpha_{j}\right)$ with $J_{\ell_{j}}\left(\overline{\alpha_{j}}\right)$, for complex nonreal $\alpha_{j}$. On the other hand, as follows from Proposition 3.4, the $\mathbb{C}$-Kronecker form of any complex hermitian pencil has the property that for every complex nonreal eigenvalue $\beta$ and every positive integer $m$ the number of blocks $t I_{m}+J_{m}(\beta)$ in the $\mathbb{C}$-Kronecker form coincides with the number of blocks $t I_{m}+J_{m}(\bar{\beta})$. Therefore, the $\mathbb{C}$-Kronecker forms of $\mathrm{i} A+t(\mathrm{i} B)$ and $\mathrm{i} A^{\prime}+t\left(\mathrm{i} B^{\prime}\right)$ must be the same. Now the direct part of (a) follows from Proposition 3.4(b).

Part (b). We need only to check that the pencils $(-\mathrm{i})\left(F_{k}+t G_{k}\right)$ and $\mathrm{i}\left(F_{k}+t G_{k}\right)$ are (IV)-congruent, and that the pencils $(-\mathrm{i})\left((t+\alpha) F_{\ell}+G_{\ell}\right)$ and $\mathrm{i}\left((t+\alpha) F_{\ell}+G_{\ell}\right)$, 
where $\alpha$ is real, are also (IV)-congruent. Indeed,

$$
\begin{aligned}
\left(-\mathrm{j} I_{k}\right)\left(\mathrm{i}\left(F_{k}+t G_{k}\right)\right)\left(\mathrm{j} I_{k}\right) & =(-\mathrm{i})\left(F_{k}+t G_{k}\right) \\
\left(-\mathrm{j} I_{k}\right)\left(\mathrm{i}\left((t+\alpha) F_{\ell}+G_{\ell}\right)\right)\left(\mathrm{j} I_{k}\right) & =(-\mathrm{i})\left((t+\alpha) F_{\ell}+G_{\ell}\right) .
\end{aligned}
$$

Part (c). Assume that $A+t B$ and $A^{\prime}+t B^{\prime}$ are $\mathbb{H}$-strictly equivalent. By part (a), the canonical forms of $\mathrm{i} A+t(\mathrm{i} B)$ and of $\mathrm{i} A^{\prime}+t\left(\mathrm{i} B^{\prime}\right)$ under (III)-congruence are given by (3.3) and (5.1), respectively. Clearly, $A+t B$ and $A^{\prime}+t B^{\prime}$ are $\mathbb{C}$-strictly equivalent. Conversely, if $A+t B$ and $A^{\prime}+t B^{\prime}$ are $\mathbb{C}$-strictly equivalent, then so are the complex hermitian pencils $\mathrm{i} A+t(\mathrm{i} B)$ and of $\mathrm{i} A^{\prime}+t\left(\mathrm{i} B^{\prime}\right)$. It follows (see $[11$, Theorem 5.1], for example) that the canonical forms of $\mathrm{i} A+t(\mathrm{i} B)$ and of $\mathrm{i} A^{\prime}+t\left(\mathrm{i} B^{\prime}\right)$ under (III)-congruence can possibly differ only in the signs in their sign characteristics. Now the result of part (b) yields the (IV)-congruence of $A+t B$ and $A^{\prime}+t B^{\prime}$.

6. Comparison of congruence: mixed hermitian - skewhermitian complex pairs. In this section we compare strict equivalences, (III)-congruences and (IV)-congruences of complex matrix pencils $A+t B$, or equivalently, pairs of complex matrices $(A, B)$, where $A$ is hermitian and $B$ is skewhermitian. We state the main results in the next subsection. The rather long proofs are relegated to Subsection 6.2.

6.1. Main results. We state our main theorem for comparison of strict equivalences:

Theorem 6.1. Let $A, A^{\prime} \in \mathbb{C}^{n \times n}$ be complex hermitian matrices and let $B, B^{\prime} \in$ $\mathbb{C}^{n \times n}$ be complex skew-hermitian matrices. Assume that the complex matrix pencils $A+t B$ and $A^{\prime}+t B^{\prime}$ are $\mathbb{H}$-strictly equivalent, and that the hermitian matrix pencil $A+t(\mathrm{i} B)$ is $\mathbb{C}$-strictly equivalent to the form (3.3), where

$$
\alpha_{1}=\alpha_{2}=\cdots=\alpha_{q^{\prime}}=0, \quad \alpha_{w} \in \mathbb{R} \backslash\{0\} \text { for } w=q^{\prime}+1, q^{\prime}+2, \ldots, q
$$

for some $q^{\prime}$ (the case $q^{\prime}=0$ is not excluded), and where we may take all signs $\delta_{j}$ 's and $\eta_{j}$ 's equal to 1 .

Then the hermitian pencil $A^{\prime}+t\left(\mathrm{i} B^{\prime}\right)$ is $\mathbb{C}$-strictly equivalent to a hermitian matrix pencil of the following form:

$$
\begin{aligned}
0_{u \times u} & \oplus\left(t\left[\begin{array}{ccc}
0 & 0 & F_{\varepsilon_{1}} \\
0 & 0 & 0 \\
F_{\varepsilon_{1}} & 0 & 0
\end{array}\right]+G_{2 \varepsilon_{1}+1}\right) \oplus \cdots \oplus\left(t\left[\begin{array}{ccc}
0 & 0 & F_{\varepsilon_{p}} \\
0 & 0 & 0 \\
F_{\varepsilon_{p}} & 0 & 0
\end{array}\right]+G_{2 \varepsilon_{p}+1}\right) \\
& \oplus\left(F_{k_{1}}+t G_{k_{1}}\right) \oplus \cdots \oplus\left(F_{k_{r}}+t G_{k_{r}}\right) \\
& \oplus\left(t F_{\ell_{1}}+G_{\ell_{1}}\right) \oplus \cdots \oplus\left(t F_{\ell_{q^{\prime}}}+G_{\ell_{q^{\prime}}}\right) \\
& \left.\oplus\left(\left(t+\kappa_{q^{\prime}+1}^{\prime} \alpha_{q^{\prime}+1}\right) F_{\ell_{q^{\prime}+1}}+G_{\ell_{q^{\prime}+1}}\right) \oplus \cdots \oplus\left(t+\kappa_{q}^{\prime} \alpha_{q}\right) F_{\ell_{q}}+G_{\ell_{q}}\right) \\
& \oplus\left(\left[\begin{array}{cc}
\left(t+\overline{\beta_{1}^{\prime}}\right) F_{m_{1}} & \left(t+\beta_{1}^{\prime}\right) F_{m_{1}} \\
0 & 0
\end{array}\right]+\left[\begin{array}{cc}
0 & G_{m_{1}} \\
G_{m_{1}} & 0
\end{array}\right]\right) \\
(6.1) \quad & \left.\left.\oplus \cdots \oplus \begin{array}{cc}
0 & G_{m_{s}}
\end{array}\right]\right),
\end{aligned}
$$


where

(6.2) for each $j=1, \ldots, s$, either $\beta_{j}^{\prime}=\beta_{j}$ or $\beta_{j}^{\prime}=-\overline{\beta_{j}}$, and $\kappa_{q^{\prime}+1}^{\prime}, \ldots, \kappa_{q}^{\prime} \in\{1,-1\}$.

Conversely, suppose that $A^{\prime}+t B^{\prime}$ and $A^{\prime \prime}+t B^{\prime \prime}$ are pencils of the form (6.1), with the parameters

$$
\left\{\kappa_{q^{\prime}+1}^{\prime}, \ldots, \kappa_{q}^{\prime} ; \beta_{1}^{\prime}, \ldots, \beta_{s}^{\prime}\right\}
$$

subject to (6.2) for $A^{\prime}+t B^{\prime}$, and with the corresponding parameters

$$
\left\{\kappa_{q^{\prime}+1}^{\prime \prime}, \ldots, \kappa_{q}^{\prime \prime} ; \beta_{1}^{\prime \prime}, \ldots, \beta_{s}^{\prime \prime}\right\},
$$

again subject to conditions analogous to (6.2), for $A^{\prime \prime}+t B^{\prime \prime}$. Then the complex hermitian-skewhermitian matrix pencils $A^{\prime}+t(-\mathrm{i}) B^{\prime}$ and $A^{\prime \prime}+t(-\mathrm{i}) B^{\prime \prime}$ are $\mathbb{H}$-strictly equivalent.

By inspection of the form (6.1), the following corollary is immediate:

Corollary 6.2. Let

$$
A, B \in \mathbb{C}^{n \times n}, \quad A=A^{*}, \quad B=-B^{*} .
$$

Then the following two statements are equivalent:

(1) Every hermitian-skewhermitian complex matrix pencil $A^{\prime}+t B^{\prime}, A^{\prime}=A^{\prime *}$, $B^{\prime}=-B^{\prime *}$ which is $\mathbb{H}$-strictly equivalent to $A+t B$ is also $\mathbb{C}$-strictly equivalent to $A+t B$

(2) All eigenvalues of $A+t B$ different from the infinity are real.

Comparing with Proposition 2.6, we see that the additional hypothesis of hermitian-skewhermitian property of complex matrix pencils does not alter the criterion for the property that $\mathbb{H}$-strict equivalence implies $\mathbb{C}$-strict equivalence.

Next, we state the main result on comparing (III)-congruences and (IV)- congruences for hermitian-skewhermitian matrix pairs.

TheOREm 6.3. Let $A, A^{\prime} \in \mathbb{C}^{n \times n}$ be complex hermitian matrices and let $B, B^{\prime} \in$ $\mathbb{C}^{n \times n}$ be complex skew-hermitian matrices. Let (3.3) be the canonical form of the hermitian pencil $A+t(\mathrm{i} B)$ under (III)-congruence, where

$$
\alpha_{1}=\alpha_{2}=\cdots=\alpha_{q^{\prime}}=0, \quad \alpha_{w} \in \mathbb{R} \backslash\{0\} \text { for } w=q^{\prime}+1, q^{\prime}+2, \ldots, q
$$

for some $q^{\prime}$ (the case $q^{\prime}=0$ is not excluded). Assume that the complex matrix pencils $A+t B$ and $A^{\prime}+t B^{\prime}$ are (IV)-congruent. Then the canonical form of the hermitian pencil $A^{\prime}+t\left(\mathrm{i} B^{\prime}\right)$ under (III)-congruence has the following structure:

$$
\begin{aligned}
0_{u \times u} & \oplus\left(t\left[\begin{array}{ccc}
0 & 0 & F_{\varepsilon_{1}} \\
0 & 0 & 0 \\
F_{\varepsilon_{1}} & 0 & 0
\end{array}\right]+G_{2 \varepsilon_{1}+1}\right) \oplus \cdots \oplus\left(t\left[\begin{array}{ccc}
0 & 0 & F_{\varepsilon_{p}} \\
0 & 0 & 0 \\
F_{\varepsilon_{p}} & 0 & 0
\end{array}\right]+G_{2 \varepsilon_{p}+1}\right) \\
& \oplus \delta_{1}^{\prime}\left(F_{k_{1}}+t G_{k_{1}}\right) \oplus \cdots \oplus \delta_{r}^{\prime}\left(F_{k_{r}}+t G_{k_{r}}\right) \\
& \oplus \eta_{1}^{\prime}\left(t F_{\ell_{1}}+G_{\ell_{1}}\right) \oplus \cdots \oplus \eta_{q^{\prime}}^{\prime}\left(t F_{\ell_{q^{\prime}}}+G_{\ell_{q^{\prime}}}\right)
\end{aligned}
$$




$$
\begin{aligned}
& \oplus \eta_{q^{\prime}+1}^{\prime}\left(\left(t+\kappa_{q^{\prime}+1}^{\prime} \alpha_{q^{\prime}+1}\right) F_{\ell_{q^{\prime}+1}}+G_{\ell_{q^{\prime}+1}}\right) \oplus \cdots \oplus \eta_{q}^{\prime}\left(\left(t+\kappa_{q}^{\prime} \alpha_{q}\right) F_{\ell_{q}}+G_{\ell_{q}}\right) \\
& \oplus\left(\left[\begin{array}{cc}
0 & \left(t+\beta_{1}^{\prime}\right) F_{m_{1}} \\
\left(t+\overline{\beta_{1}^{\prime}}\right) F_{m_{1}} & 0
\end{array}\right]+\left[\begin{array}{cc}
0 & G_{m_{1}} \\
G_{m_{1}} & 0
\end{array}\right]\right) \\
& \oplus \cdots \oplus\left(\left[\begin{array}{cc}
0 & \left(t+\beta_{s}^{\prime}\right) F_{m_{s}} \\
\left(t+\frac{\beta_{s}^{\prime}}{s}\right) F_{m_{s}} & 0
\end{array}\right]+\left[\begin{array}{cc}
0 & G_{m_{s}} \\
G_{m_{s}} & 0
\end{array}\right]\right),
\end{aligned}
$$

where for each $j=1, \ldots, s$, either $\beta_{j}^{\prime}=\beta_{j}$ or $\beta_{j}^{\prime}=-\overline{\beta_{j}}$, and $\delta_{1}^{\prime}, \ldots, \delta_{r}^{\prime}, \eta_{1}^{\prime}, \ldots, \eta_{q}^{\prime}$, and $\kappa_{q^{\prime}+1}^{\prime}, \ldots, \kappa_{q}^{\prime}$ are signs \pm 1 subject to the following restrictions (1), (2), and (3):

(1) $\delta_{j}^{\prime}=\delta_{j}$ if $k_{j}$ is odd $(j=1,2, \ldots, r)$,

(2) $\eta_{w}^{\prime}=\eta_{w}$ if $\ell_{w}$ is even $\left(w=1,2, \ldots, q^{\prime}\right)$,

(3) for every nonzero real eigenvalue $\alpha$ of $A+t(\mathrm{i} B)$ and for every index $\ell$ of $\alpha$, the following holds: if $w_{1}, \ldots, w_{k}$ are the distinct integers between $q^{\prime}+1$ and $q$ such that $\alpha_{w_{j}}=\alpha$ and $\ell_{w_{j}}=\ell, j=1,2, \ldots, k$, then there exists a permutation $\lambda$ of $\{1,2, \ldots, k\}$ such that the integers $\ell+\frac{1}{2}\left(\left(1+\kappa_{w_{\lambda(j)}}^{\prime}\right) \ell+\eta_{w_{\lambda(j)}}^{\prime}-\eta_{w_{j}}\right)$ are even for $j=1,2, \ldots, k$.

Conversely, suppose that $A^{\prime}+t B^{\prime}$ and $A^{\prime \prime}+t B^{\prime \prime}$ are pencils of the form (6.3), with the parameters

$$
\left\{\delta_{1}^{\prime}, \ldots, \delta_{r}^{\prime} ; \eta_{1}^{\prime}, \ldots, \eta_{q}^{\prime} ; \kappa_{q^{\prime}+1}^{\prime}, \ldots, \kappa_{q}^{\prime} ; \beta_{1}^{\prime}, \ldots, \beta_{s}^{\prime}\right\}
$$

for $A^{\prime}+t B^{\prime}$ (subject to the restrictions (1), (2), and (3)), and with the corresponding parameters

$$
\left\{\delta_{1}^{\prime \prime}, \ldots, \delta_{r}^{\prime \prime} ; \eta_{1}^{\prime \prime}, \ldots, \eta_{q}^{\prime \prime} ; \kappa_{q^{\prime}+1}^{\prime \prime}, \ldots, \kappa_{q}^{\prime \prime} ; \beta_{1}^{\prime \prime}, \ldots, \beta_{s}^{\prime \prime}\right\}, \quad \delta_{j}^{\prime \prime}, \eta_{k}^{\prime \prime}, \kappa_{\ell}^{\prime \prime} \in\{1,-1\},
$$

for $A^{\prime \prime}+t B^{\prime \prime}$ subject to the conditions:

(a) $\delta_{j}^{\prime \prime}=\delta_{j}$ if $k_{j}$ is odd $(j=1,2, \ldots, r)$;

(b) $\eta_{w}^{\prime \prime}=\eta_{w}$ if $\ell_{w}$ is even $\left(w=1,2, \ldots, q^{\prime}\right)$;

(c) for every nonzero real eigenvalue $\alpha$ of $A+t(\mathrm{i} B)$ and for every index $\ell$ of $\alpha$, the following holds: if $w_{1}, \ldots, w_{k}$ are the distinct integers between $q^{\prime}+1$ and $q$ such that $\alpha_{w_{j}}=\alpha$ and $\ell_{w_{j}}=\ell, j=1,2, \ldots, k$, then there exists a permutation $\lambda$ of $\{1,2, \ldots, k\}$ such that the integers $\ell+\frac{1}{2}\left(\left(1+\kappa_{w_{\lambda(j)}}^{\prime \prime}\right) \ell+\eta_{w_{\lambda(j)}}^{\prime \prime}-\eta_{w_{j}}\right)$ are even for $j=1,2, \ldots, k$.

(d) for each $j=1, \ldots, s$, either $\beta_{j}^{\prime \prime}=\beta_{j}$ or $\beta_{j}^{\prime \prime}=-\overline{\beta_{j}}$.

Then the complex hermitian-skewhermitian matrix pencils $A^{\prime}+t(-\mathrm{i}) B^{\prime}$ and $A^{\prime \prime}+$ $t(-\mathrm{i}) B^{\prime \prime}$ are (IV)-congruent.

The following corollary is noteworthy; its proof is immediate from Theorem 6.3.

Corollary 6.4. The following two statements are equivalent for a complex hermitian pencil $A+t B, A=A^{*}, B=B^{*} \in \mathbb{C}^{n \times n}$ :

(1) For every complex hermitian pencil $A^{\prime}+t B^{\prime}$, the pencils $A^{\prime}+t B^{\prime}$ and $A+t B$ are (III)-congruent if and only if the hermitian-skewhermitian pencils $A^{\prime}+$ $t(-\mathrm{i}) B^{\prime}$ and $A+t(-\mathrm{i}) B$ are (IV)-congruent.

(2) All eigenvalues of $A+t B$ different from infinity are real, and in addition, in the complex Kronecker form of $A+t B$ there are no even multiplicities corresponding to the eigenvalue at infinity and there are no odd multiplicities corresponding to the eigenvalue zero. 
It is interesting to compare to Corollary 6.2: In contrast to the strict equivalences comparison in Corollary 6.2, the congruences comparison in Corollary 6.4 involves additional hypotheses concerning the eigenvalues at zero and at infinity.

6.2. Proofs of Theorems 6.1 and 6.3. It will be convenient to collect separately several facts that will be used in the proof:

Lemma 6.5. In the statements $(\mathrm{C})-(\mathrm{G})$ below, $\eta, \eta^{\prime}, \kappa^{\prime}$ are signs \pm 1 and $\alpha$ is a nonzero real number. We have:

(A) $F_{k}+t(-\mathrm{i}) G_{k}$ is not (IV)-congruent to $-F_{k}+t \mathrm{i} G_{k}$ for odd $k$;

(A') $F_{k}+t(-\mathrm{i}) G_{k}$ is (IV)-congruent to $-F_{k}+t \mathrm{i} G_{k}$ for even $k$;

(B) $t(-\mathrm{i}) F_{\ell}+G_{\ell}$ is not (IV)-congruent to $\mathrm{ti} F_{\ell}-G_{\ell}$ for even $\ell$;

(B') $t(-\mathrm{i}) F_{\ell}+G_{\ell}$ is (IV)-congruent to $\mathrm{i} F_{\ell}-G_{\ell}$ for odd $\ell$;

(C) For $\eta^{\prime} \neq \eta$ and $\kappa^{\prime}=1$, the pencil

$$
\eta^{\prime}\left(\left(-i t+\kappa^{\prime} \alpha\right) F_{\ell}+G_{\ell}\right)
$$

is not (IV)-congruent to

$$
\eta\left((-i t+\alpha) F_{\ell}+G_{\ell}\right) ;
$$

(D) For $\kappa^{\prime}=-1, \ell$ even, and $\eta^{\prime}=\eta$, the pencil (6.4) is (IV)-congruent to (6.5);

(E) For $\kappa^{\prime}=-1, \ell$ even, and $\eta^{\prime}=-\eta$, the pencil (6.4) is not (IV)-congruent to $(6.5)$;

(F) For $\kappa^{\prime}=-1, \ell$ odd and $\eta^{\prime}=-\eta$, the pencil (6.4) is (IV)-congruent to (6.5);

(G) For $\kappa^{\prime}=-1, \ell$ odd and $\eta^{\prime}=\eta,(6.4)$ is not (IV)-congruent to (6.5);

(H) If $\beta \in \mathbb{C} \backslash \mathbb{R}$, then the matrix pencil

$$
\left[\begin{array}{cc}
0 & (-\mathrm{i} t+\beta) F_{m}+G_{m} \\
(-\mathrm{i} t+\bar{\beta}) F_{m}+G_{m} & 0
\end{array}\right]
$$

is (IV)-congruent to

$$
\left[\begin{array}{cc}
0 & (-\mathrm{i} t-\bar{\beta}) F_{m}+G_{m} \\
(-\mathrm{i} t-\beta) F_{m}+G_{m} & 0
\end{array}\right]
$$

Proof. Statements (A) and (B) are contained in [18, Lemma 5.5(2),(4)]. Statements $\left(\mathrm{A}^{\prime}\right)$ and $\left(\mathrm{B}^{\prime}\right)$ follow from the equalities

$$
\operatorname{diag}(\mathrm{j},-\mathrm{j}, \ldots, \mathrm{j},-\mathrm{j})\left(F_{k}+t \mathrm{i} G_{k}\right) \operatorname{diag}(-\mathrm{j}, \mathrm{j}, \ldots,-\mathrm{j}, \mathrm{j})=-\left(F_{k}+t \mathrm{i} G_{k}\right)
$$

for even $k$ and

$$
\operatorname{diag}(\mathrm{j},-\mathrm{j}, \ldots,-\mathrm{j}, \mathrm{j})\left(G_{\ell}+t \mathrm{i} F_{\ell}\right) \operatorname{diag}(-\mathrm{j}, \mathrm{j}, \ldots, \mathrm{j},-\mathrm{j})=-\left(G_{\ell}+t \mathrm{i} F_{\ell}\right)
$$

for odd $\ell$. Statement (C) is a consequence of [18, Lemma 5.5(5), Theorem 5.3]; indeed, it follows from these results that any matrix pencil of mixed hermitian-skewhermitian quaternionic matrices whose $\mathbb{H}$-Kronecker form consists of exactly one Jordan block and the eigenvalue of this block is nonzero with zero real part, is not (IV)-congruent 
to its negative. For the statement (D) note the equality (taking $\eta^{\prime}=\eta=1$ without loss of generality)

$$
\operatorname{diag}(-\mathrm{j}, \mathrm{j}, \ldots,-\mathrm{j}, \mathrm{j})\left((-\mathrm{i} t-\alpha) F_{\ell}+G_{\ell}\right) \operatorname{diag}(\mathrm{j},-\mathrm{j}, \ldots, \mathrm{j},-\mathrm{j})=\left((-\mathrm{i} t+\alpha) F_{\ell}+G_{\ell}\right),
$$

where $\alpha \in \mathbb{R} \backslash\{0\}$ and $\ell$ is even.

Consider (E). By the already proved statement (D), the matrix pencils

$$
\eta^{\prime}\left((-\mathrm{i} t-\alpha) F_{\ell}+G_{\ell}\right) \quad \text { and } \quad \eta^{\prime}\left((-\mathrm{i} t+\alpha) F_{\ell}+G_{\ell}\right)
$$

are (IV)-congruent; but

$$
\eta^{\prime}\left((-\mathrm{i} t+\alpha) F_{\ell}+G_{\ell}\right)=-\eta\left((-\mathrm{i} t+\alpha) F_{\ell}+G_{\ell}\right),
$$

which is not (IV)-congruent to (6.5) by statement (C). Statement (F) is proved by the equality

$(\operatorname{diag}(\mathrm{j},-\mathrm{j}, \mathrm{j}, \ldots,-\mathrm{j}, \mathrm{j}))\left((\mathrm{it}+\alpha) F_{\ell}-G_{\ell}\right)(\operatorname{diag}(-\mathrm{j}, \mathrm{j},-\mathrm{j}, \ldots, \mathrm{j},-\mathrm{j}))=(-\mathrm{i} t+\alpha) F_{\ell}+G_{\ell}$,

where $\ell$ is odd. For the proof of $(\mathrm{G})$ observe that the real symmetric matrices $\eta^{\prime} \kappa^{\prime} \alpha F_{\ell}+$ $\eta^{\prime} G_{\ell}$ and $\eta \alpha F_{\ell}+\eta G_{\ell}$ (the values of the pencils (6.4) and (6.5) when $t=0$ ) have different inertia, therefore these two matrices cannot be (IV)-congruent. For the statement $(\mathrm{H})$, let $\alpha$ and $\gamma$ be nonzero quaternions satisfying the properties

$$
\alpha(-\mathrm{i}) \alpha^{-1}=\mathrm{i}, \quad \alpha(-1)^{m} \gamma^{*}=1,
$$

and let

$$
X:=\left(\operatorname{diag}\left(\alpha,-\alpha, \ldots,(-1)^{m-1} \alpha\right)\right) \oplus\left(\operatorname{diag}\left(\gamma,-\gamma, \ldots,(-1)^{m-1} \gamma\right)\right) \in \mathbb{H}^{2 m \times 2 m} .
$$

Then a straightforward computation shows that

$$
\begin{gathered}
X\left[\begin{array}{cc}
0 & (-\mathrm{i} t+\beta) F_{m}+G_{m} \\
(-\mathrm{i} t+\bar{\beta}) F_{m}+G_{m} & 0
\end{array}\right] X^{*}= \\
{\left[\begin{array}{cc}
0 & (-\mathrm{i} t-\bar{\beta}) F_{m}+G_{m} \\
(-\mathrm{i} t-\beta) F_{m}+G_{m} & 0
\end{array}\right] .}
\end{gathered}
$$

This completes the proof of Lemma 6.5.

Statements (C) through $(\mathrm{G})$ of Lemma 6.5 can be conveniently summarized as follows.

Corollary 6.6. For signs $\eta, \eta^{\prime}, \kappa^{\prime} \in\{1,-1\}$, positive integer $\ell$, and $\alpha \in \mathbb{R} \backslash\{0\}$, the matrix pencils (6.4) and (6.5) are (IV)-congruent if and only if the integer

$$
\ell+\frac{1}{2}\left(\left(1+\kappa^{\prime}\right) \ell+\eta^{\prime}-\eta\right)
$$


is even.

Proof of Theorem 6.3. The direct statement: The pencils $A+t B$ and $A^{\prime}+t B^{\prime}$ clearly have the same $\mathbb{H}-$ Kronecker form. Therefore, denoting by $A_{0}^{\prime}+t B_{0}^{\prime}$ the $\mathbb{C}$ Kronecker form of $A^{\prime}+t B^{\prime}$, and denoting by $A_{0}+t B_{0}$ the $\mathbb{C}$-Kronecker form of $A+t B$, we obtain in view of Proposition 2.6 that $A_{0}^{\prime}+t B_{0}^{\prime}$ is obtained from $A_{0}+t B_{0}$ by replacing some blocks $J_{\ell_{j}}\left(\alpha_{j}\right)$ with $J_{\ell_{j}}\left(\overline{\alpha_{j}}\right)$, for nonreal $\alpha_{j}$. Note that $A_{0}^{\prime}+t\left(\mathrm{i} B_{0}^{\prime}\right)$ is $\mathbb{C}$-strictly equivalent to $A^{\prime}+t\left(\mathrm{i} B^{\prime}\right)$, whereas $A_{0}+t\left(\mathrm{i} B_{0}\right)$ is $\mathbb{C}$-strictly equivalent to $A+t(\mathrm{i} B)$. Note also that

$$
\begin{gathered}
x=y+\mathrm{i} z, y, z \in \mathbb{R}, \text { is an eigenvalue of } A_{0}+t\left(\mathrm{i} B_{0}\right) \Longleftrightarrow \\
\mathrm{i} y-z \text { is an eigenvalue of } A_{0}+t B_{0} \Longleftrightarrow \\
\mathrm{i} y-z \text { and } / \text { or }-\mathrm{i} y-z \text { is an eigenvalue of } A_{0}^{\prime}+t B_{0}^{\prime} \Longleftrightarrow \\
-y+\mathrm{i} z \text { and/or } y+\mathrm{i} z \text { is an eigenvalue of } A_{0}^{\prime}+t\left(\mathrm{i} B_{0}^{\prime}\right) .
\end{gathered}
$$

Taking into account Proposition 3.4 we see that the canonical form of the complex hermitian pencil $A^{\prime}+t\left(\mathrm{i} B^{\prime}\right)$ under (III)-congruence must have the following structure:

$$
\begin{aligned}
0_{u \times u} & \left.\oplus t\left[\begin{array}{ccc}
0 & 0 & F_{\varepsilon_{1}} \\
0 & 0 & 0 \\
F_{\varepsilon_{1}} & 0 & 0
\end{array}\right]+G_{2 \varepsilon_{1}+1}\right) \oplus \cdots \oplus\left(t\left[\begin{array}{ccc}
0 & 0 & F_{\varepsilon_{p}} \\
0 & 0 & 0 \\
F_{\varepsilon_{p}} & 0 & 0
\end{array}\right]+G_{2 \varepsilon_{p}+1}\right) \\
& \oplus \delta_{1}^{\prime}\left(F_{k_{1}}+t G_{k_{1}}\right) \oplus \cdots \oplus \delta_{r}^{\prime}\left(F_{k_{r}}+t G_{k_{r}}\right) \\
& \oplus \eta_{1}^{\prime}\left(t F_{\ell_{1}}+G_{\ell_{1}}\right) \oplus \cdots \oplus \eta_{q^{\prime}}^{\prime}\left(t F_{\ell_{q^{\prime}}}+G_{\ell_{q^{\prime}}}\right) \\
& \oplus \eta_{q^{\prime}+1}^{\prime}\left(\left(t+\eta_{q^{\prime}+1}^{\prime \prime} \alpha_{q^{\prime}+1}\right) F_{\ell_{q^{\prime}+1}}+G_{\ell_{q^{\prime}+1}}\right) \oplus \cdots \oplus \eta_{q}^{\prime}\left(\left(t+\eta_{q}^{\prime \prime} \alpha_{q}\right) F_{\ell_{q}}+G_{\ell_{q}}\right) \\
& \oplus\left(\left[\begin{array}{cc}
0 & \left(t+\beta_{1}^{\prime}\right) F_{m_{1}}
\end{array}\right]+\left[\begin{array}{cc}
0 & G_{m_{1}} \\
G_{m_{1}} & 0
\end{array}\right]\right) \\
\text { (6.6) } & \oplus \cdots \oplus\left(\left[\begin{array}{cc}
0 & 0
\end{array}\right]+\left[\begin{array}{cc}
0 & G_{m_{s}} \\
G_{m_{s}} & 0
\end{array}\right]\right),
\end{aligned}
$$

where for each $j=1, \ldots, s$, either $\beta_{j}^{\prime}=\beta_{j}$ or $\beta_{j}^{\prime}=-\overline{\beta_{j}}$, and

$$
\delta_{1}^{\prime}, \ldots, \delta_{r}^{\prime}, \quad \eta_{1}^{\prime}, \ldots, \eta_{q}^{\prime}, \eta_{q^{\prime}+1}^{\prime \prime}, \ldots, \eta_{q}^{\prime \prime}
$$

are signs \pm 1 . Denote by $A_{1}+t B_{1}$ the hermitian pencil (3.3), and by $A_{1}^{\prime}+t B_{1}^{\prime}$ the hermitian pencil (6.6). Then the pencils $A_{1}+t(-\mathrm{i}) B_{1}$ and $A_{1}^{\prime}+t(-\mathrm{i}) B_{1}^{\prime}$ are (IV)congruent. In view of the canonical form for hermitian-skewhermitian quaternionic pencils under (IV)-congruence (the canonical form describes the primitive blocks for such pencils, see, for example, [18, Theorem 5.3]), the direct statement of Theorem 6.3 follows from Lemma $6.5(\mathrm{~A}),(\mathrm{B}),(\mathrm{H})$, and from Corollary 6.6. 
The converse statement follows by analogous arguments using Lemma $6.5\left(\mathrm{~A}^{\prime}\right)$, $\left(\mathrm{B}^{\prime}\right)$ and Corollary 6.6 again.

Proof of Theorem 6.1. The direct statement: Arguing as in the proof of the direct statement of Theorem 6.3, we see that the canonical form of the complex hermitian pencil $A^{\prime}+t\left(\mathrm{i} B^{\prime}\right)$ under (III)-congruence must have the structure as in (6.6). Evidently, (6.6) is $\mathbb{C}$-strictly equivalent to (6.1).

The converse statement: The $\mathbb{H}$-strict equivalence of $A^{\prime}+t(-\mathrm{i}) B^{\prime}$ and $A^{\prime \prime}+t(-\mathrm{i}) B^{\prime \prime}$ follows by observing that every pair of corresponding constituent blocks in $A^{\prime}+t(-\mathrm{i}) B^{\prime}$ and $A^{\prime \prime}+t(-\mathrm{i}) B^{\prime \prime}$ has the same $\mathbb{H}-$ Kronecker form.

7. Congruences of pairs of complex hermitian or skewhermitian matrices II. In this section we study comparison of (III)-congruence and congruences over the quaternions for matrix pencils $A+t B$, where $A= \pm A^{*}$ and $B= \pm B^{*}$ are complex matrices. As in Section 5, in all cases, we use the canonical form for (III)-congruence of complex hermitian matrix pencils given in Proposition 3.4. To compare with congruence over the quaternions, in contrast to Sections 5 and 6 , the

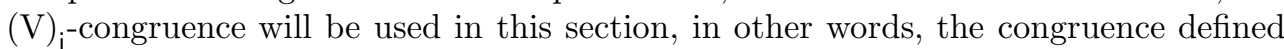
by transformations $A+t B \mapsto S^{\sigma}(A+t B) S$ with invertible quaternionic matrix $A$, where a nonstandard iaa $\sigma$ is determined by the property that $\sigma(\mathrm{i})=-\mathrm{i}$; in fact, a nonstandard iaa with this property is unique.

We clearly have $A^{*}=A^{\sigma}$ for every complex matrix $A$. Thus:

Proposition 7.1. If complex matrix pencils are (III)-congruent, then they are $(\mathrm{V})_{\mathrm{i}}$-congruent. We explore in this section to what extent the converse is true, for complex matrix pencils with symmetries.

7.1. Hermitian and skewhermitian matrix pencils. We start with complex hermitian matrix pencils:

Theorem 7.2. Let $A+t B$ and $A^{\prime}+t B^{\prime}$ be complex matrix pencils, with hermitian matrices $A, A^{\prime}, B, B^{\prime}$. Then the following statements are equivalent:

(a) $A+t B$ and $A^{\prime}+t B^{\prime}$ are $\mathbb{H}$-strictly equivalent;

(b) $A+t B$ and $A^{\prime}+t B^{\prime}$ are $\mathbb{C}$-strictly equivalent;

(c) $A+t B$ and $A^{\prime}+t B^{\prime}$ are $(\mathrm{V})_{\mathrm{i}}$-congruent;

(d) The canonical forms of $A+t B$ and $A^{\prime}+t B^{\prime}$ under (III)-congruence (Proposition 3.4) may differ only in the sum of signs corresponding to any real eigenvalue (or the eigenvalue at infinity) in their respective sign characteristic.

Proof. The equivalence of (a) and (c) follows from Theorem 7.1 in [16]. By combining Propositions 3.4(b) and 2.6 we see that (a) and (b) are equivalent. Finally, (b) and (d) are equivalent in view of Proposition 3.4(b) again.

Next, consider skewhermitian complex matrix pencils.

TheOREM 7.3. Let $A+t B$ and $A^{\prime}+t B^{\prime}$ be complex matrix pencils, with skewhermitian $m \times m$ matrices $A, A^{\prime}, B, B^{\prime}$. Then:

(a) The pencils $A+t B$ and $A^{\prime}+t B^{\prime}$ are $\mathbb{C}$-strictly equivalent if and only if they are $\mathbb{H}$-strictly equivalent. 
(b) The pencils $A+t B$ and $A^{\prime}+t B^{\prime}$ are (III)-congruent if and only if they are $(\mathrm{V})_{\mathrm{i}}$-congruent.

Proof. Part (a): Note that in view of Proposition 3.4, the $\mathbb{C}$-Kronecker forms of $A+t B$ and of $A^{\prime}+t B^{\prime}$, which are the same as the $\mathbb{C}$-Kronecker forms of complex hermitian matrix pencils $\mathrm{i} A+t \mathrm{i} B$ and $\mathrm{i} A^{\prime}+t \mathrm{i} B^{\prime}$, respectively, are symmetric relative to the real axis: If $\alpha \in \mathbb{C}$ is a nonreal eigenvalue of $A+t B$, then so is $\bar{\alpha}$, and the indices of $\alpha$ coincide with the indices of $\bar{\alpha}$; an analogous statement holds for $A^{\prime}+t B^{\prime}$. In view of Proposition 2.6 it follows that if the pencils $A+t B$ and $A^{\prime}+t B^{\prime}$ are $\mathbb{H}$-strictly equivalent then they are also $\mathbb{C}$-strictly equivalent.

Part (b): Let $A+t B$ and $A^{\prime}+t B^{\prime}$ be $(\mathrm{V})_{\mathrm{i}}$-congruent. Then in particular the pencils are $\mathbb{H}$-strictly equivalent, hence by the part (a) also $\mathbb{C}$-strictly equivalent. Therefore, the hermitian matrix pencils $-\mathrm{i} A+t(-\mathrm{i}) B$ and $-\mathrm{i} A^{\prime}+t(-\mathrm{i}) B^{\prime}$ are $\mathbb{C}$-strictly equivalent as well. Arguing by contradiction, and assuming that $-\mathrm{i} A+t(-\mathrm{i}) B$ and $-\mathrm{i} A^{\prime}+t(-\mathrm{i}) B^{\prime}$ are not (III)-congruent, we obtain (by using the canonical form of Proposition 3.4 for complex hermitian matrix pencils $-\mathrm{i} A+t(-\mathrm{i}) B$ and $\left.-\mathrm{i} A^{\prime}+t(-\mathrm{i}) B^{\prime}\right)$ that there exist invertible complex matrices $S$ and $T$ with the following properties:

$$
\begin{aligned}
& S^{*}(A+t B) S=0_{u \times u} \oplus \bigoplus_{j=1}^{p}\left(t \mathrm{i}\left[\begin{array}{ccc}
0 & 0 & F_{\varepsilon_{j}} \\
0 & 0 & 0 \\
F_{\varepsilon_{j}} & 0 & 0
\end{array}\right]+\mathrm{i} G_{2 \varepsilon_{j}+1}\right) \\
& \oplus \bigoplus_{j=1}^{r}\left(\delta_{j}\left(\mathrm{i} F_{k_{j}}+t \mathrm{i} G_{k_{j}}\right)\right) \oplus \bigoplus_{j=1}^{q}\left(\eta_{j}\left(\left(t+\alpha_{j}\right) \mathrm{i} F_{\ell_{j}}+\mathrm{i} G_{\ell_{j}}\right)\right) \\
& \oplus \bigoplus_{j=1}^{s}\left(\left[\begin{array}{cc}
0 & \left(t+\beta_{j}\right) \mathrm{i} F_{m_{j}} \\
\left(t+\bar{\beta}_{j}\right) \mathrm{i} F_{m_{j}} & 0
\end{array}\right]+\left[\begin{array}{cc}
0 & \mathrm{i} G_{m_{j}} \\
\mathrm{i} G_{m_{j}} & 0
\end{array}\right]\right) ; \\
& T^{*}\left(A^{\prime}+t B^{\prime}\right) T=0_{u \times u} \oplus \bigoplus_{j=1}^{p}\left(t \mathrm{i}\left[\begin{array}{ccc}
0 & 0 & F_{\varepsilon_{j}} \\
0 & 0 & 0 \\
F_{\varepsilon_{j}} & 0 & 0
\end{array}\right]+\mathrm{i} G_{2 \varepsilon_{j}+1}\right) \\
& \oplus \bigoplus_{j=1}^{r}\left(\delta_{j}^{\prime}\left(\mathrm{i} F_{k_{j}}+t \mathrm{i} G_{k_{j}}\right)\right) \oplus \bigoplus_{j=1}^{q}\left(\eta_{j}^{\prime}\left(\left(t+\alpha_{j}\right) \mathrm{i} F_{\ell_{j}}+\mathrm{i} G_{\ell_{j}}\right)\right) \\
& \oplus \bigoplus_{j=1}^{s}\left(\left[\begin{array}{cc}
0 & \left(t+\beta_{j}\right) \mathrm{i} F_{m_{j}} \\
\left(t+\bar{\beta}_{j}\right) \mathrm{i} F_{m_{j}} & 0
\end{array}\right]+\left[\begin{array}{cc}
0 & \mathrm{i} G_{m_{j}} \\
\mathrm{i} G_{m_{j}} & 0
\end{array}\right]\right) ;
\end{aligned}
$$

where in (7.1) and (7.2), $\varepsilon_{1} \leq \cdots \leq \varepsilon_{p}$ and $k_{1} \leq \cdots \leq k_{r}$ are positive integers, $\alpha_{j}$ are real numbers, $\beta_{j}$ are complex nonreal numbers with positive imaginary parts,

$$
\delta_{1}, \ldots, \delta_{r}, \quad \eta_{1}, \ldots, \eta_{q}, \quad \delta_{1}^{\prime}, \ldots, \delta_{r}^{\prime}, \quad \eta_{1}^{\prime}, \ldots, \eta_{q}^{\prime}
$$

are signs, each equal to +1 or -1 , and in addition we have

$$
\delta_{1}+\cdots+\delta_{r} \neq \delta_{1}^{\prime}+\cdots+\delta_{r}^{\prime}
$$


or there exists a real number $\alpha$ such that

$$
\sum_{\left\{j: \alpha_{j}=\alpha\right\}} \eta_{j} \neq \sum_{\left\{j: \alpha_{j}=\alpha\right\}} \eta_{j}^{\prime}
$$

(Equalities (7.3) and (7.4) express the assumption that the pencils $-\mathrm{i} A+t(-\mathrm{i}) B$ and $-\mathrm{i} A^{\prime}+t(-\mathrm{i}) B^{\prime}$ are not (III)-congruent.) Now a comparison with the canonical form of Theorem 8.1 of [16] for $\sigma$-skewsymmetric quaternionic matrix pencils shows that the right hand sides of $(7.1)$ and $(7.2)$ are not $(\mathrm{V})_{\mathrm{i}}$-congruent, a contradiction with the $(\mathrm{V})_{\mathrm{i}}$-congruence of $A+t B$ and $A^{\prime}+t B^{\prime}$.

7.2. Mixed hermitian-skewhermitian matrix pencils. We consider here in a separate subsection the more difficult case of mixed hermitian-skewhermitian complex matrix pencils. Thus, the pencils under consideration will be of the form $A+$ $t B$, where $A$ is a complex hermitian matrix, and $B$ is a complex skewhermitian matrix. As in the preceding subsection, we compare (III)-congruence and (V) $)^{-}$-congruence of such pencils, where the $(\mathrm{V})_{\mathrm{i}}$-congruence is determined by the nonstandard iaa $\sigma$ such that $\sigma(\mathrm{i})=-\mathrm{i}$.

The main result and its corollary are parallel to Theorem 6.3 and Corollary 6.4 :

TheOREM 7.4. Let $A, A^{\prime} \in \mathbb{C}^{n \times n}$ be complex hermitian matrices and let $B, B^{\prime} \in$ $\mathbb{C}^{n \times n}$ be complex skew-hermitian matrices. Let (3.3) be the canonical form of the hermitian pencil $A+t(\mathrm{i} B)$ under (III)-congruence, where

$$
\alpha_{1}=\alpha_{2}=\cdots=\alpha_{q^{\prime}}=0, \quad \alpha_{w} \in \mathbb{R} \backslash\{0\} \text { for } w=q^{\prime}+1, q^{\prime}+2, \ldots, q
$$

for some $q^{\prime}$ (the case $q^{\prime}=0$ is not excluded). Assume that the complex matrix pencils $A+t B$ and $A^{\prime}+t B^{\prime}$ are $(\mathrm{V})_{\mathrm{i}}$-congruent. Then the canonical form of the hermitian pencil $A^{\prime}+t\left(\mathrm{i} B^{\prime}\right)$ under (III)-congruence has the following structure:

$$
\begin{aligned}
0_{u \times u} & \oplus\left(t\left[\begin{array}{ccc}
0 & 0 & F_{\varepsilon_{1}} \\
0 & 0 & 0 \\
F_{\varepsilon_{1}} & 0 & 0
\end{array}\right]+G_{2 \varepsilon_{1}+1}\right) \oplus \cdots \oplus\left(t\left[\begin{array}{ccc}
0 & 0 & F_{\varepsilon_{p}} \\
0 & 0 & 0 \\
F_{\varepsilon_{p}} & 0 & 0
\end{array}\right]+G_{2 \varepsilon_{p}+1}\right) \\
& \oplus \delta_{1}^{\prime}\left(F_{k_{1}}+t G_{k_{1}}\right) \oplus \cdots \oplus \delta_{r}^{\prime}\left(F_{k_{r}}+t G_{k_{r}}\right) \\
& \oplus \eta_{1}^{\prime}\left(t F_{\ell_{1}}+G_{\ell_{1}}\right) \oplus \cdots \oplus \eta_{q^{\prime}}^{\prime}\left(t F_{\ell_{q^{\prime}}}+G_{\ell_{q^{\prime}}}\right) \\
& \oplus \eta_{q^{\prime}+1}^{\prime}\left(\left(t+\kappa_{q^{\prime}+1}^{\prime} \alpha_{q^{\prime}+1}\right) F_{\ell_{q^{\prime}+1}}+G_{\ell_{q^{\prime}+1}}\right) \oplus \cdots \oplus \eta_{q}^{\prime}\left(\left(t+\kappa_{q}^{\prime} \alpha_{q}\right) F_{\ell_{q}}+G_{\ell_{q}}\right) \\
& \oplus\left(\left[\begin{array}{cc}
0 & \left(t+\beta_{1}^{\prime}\right) F_{m_{1}} \\
\left(t+\frac{\beta_{1}^{\prime}}{)} F_{m_{1}}\right. & 0
\end{array}\right]+\left[\begin{array}{cc}
0 & G_{m_{1}} \\
G_{m_{1}} & 0
\end{array}\right]\right) \\
(7.5) & \oplus \cdots \oplus\left(\left[\begin{array}{cc}
0 & \left(t+\beta_{s}^{\prime}\right) F_{m_{s}} \\
\left(t+\frac{\beta_{s}^{\prime}}{0}\right) F_{m_{s}} & 0
\end{array}\right]+\left[\begin{array}{cc}
0 & G_{m_{s}} \\
G_{m_{s}} & 0
\end{array}\right]\right),
\end{aligned}
$$

where for each $j=1, \ldots, s$, either $\beta_{j}^{\prime}=\beta_{j}$ or $\beta_{j}^{\prime}=-\overline{\beta_{j}}$, and $\delta_{1}^{\prime}, \ldots, \delta_{r}^{\prime}, \eta_{1}^{\prime}, \ldots, \eta_{q}^{\prime}$, and $\kappa_{q^{\prime}+1}^{\prime}, \ldots, \kappa_{q}^{\prime}$ are signs \pm 1 subject to the following restrictions (1), (2), and (3):

(1) $\delta_{j}^{\prime}=\delta_{j}$ if $k_{j}$ is even $(j=1,2, \ldots, r)$,

(2) $\eta_{w}^{\prime}=\eta_{w}$ if $\ell_{w}$ is odd $\left(w=1,2, \ldots, q^{\prime}\right)$, 
(3) for every nonzero real eigenvalue $\alpha$ of $A+t(\mathrm{i} B)$ and for every index $\ell$ of $\alpha$, the following holds: if $w_{1}, \ldots, w_{k}$ are the distinct integers between $q^{\prime}+1$ and $q$ such that $\alpha_{w_{j}}=\alpha$ and $\ell_{w_{j}}=\ell, j=1,2, \ldots, k$, then there exists a permutation $\lambda$ of $\{1,2, \ldots, k\}$ with the property that the integers $\ell+\frac{1}{2}((1+$ $\left.\left.\kappa_{w_{\lambda(j)}}^{\prime}\right)(\ell+1)+\eta_{w_{\lambda(j)}}^{\prime}-\eta_{w_{j}}\right)$ are odd for $j=1,2, \ldots, k$.

Conversely, suppose that hermitian-skewhermitian matrix pencils $A^{\prime}+t B^{\prime}$ and $A^{\prime \prime}+t B^{\prime \prime}$ are of the form (6.3), with the parameters

$$
\left\{\delta_{1}^{\prime}, \ldots, \delta_{r}^{\prime} ; \eta_{1}^{\prime}, \ldots, \eta_{q}^{\prime} ; \kappa_{q^{\prime}+1}^{\prime}, \ldots, \kappa_{q}^{\prime} ; \beta_{1}^{\prime}, \ldots, \beta_{s}^{\prime}\right\}
$$

for $A^{\prime}+t B^{\prime}$ (subject to the restrictions (1), (2), and (3)), and with the corresponding parameters

$$
\left\{\delta_{1}^{\prime \prime}, \ldots, \delta_{r}^{\prime \prime} ; \eta_{1}^{\prime \prime}, \ldots, \eta_{q}^{\prime \prime} ; \kappa_{q^{\prime}+1}^{\prime \prime}, \ldots, \kappa_{q}^{\prime \prime} ; \beta_{1}^{\prime \prime}, \ldots, \beta_{s}^{\prime \prime}\right\}, \quad \delta_{j}^{\prime \prime}, \eta_{k}^{\prime \prime}, \kappa_{\ell}^{\prime \prime} \in\{1,-1\},
$$

for $A^{\prime \prime}+t B^{\prime \prime}$ subject to the conditions:

(a) $\delta_{j}^{\prime \prime}=\delta_{j}$ if $k_{j}$ is even $(j=1,2, \ldots, r)$;

(b) $\eta_{w}^{\prime \prime}=\eta_{w}$ if $\ell_{w}$ is odd $\left(w=1,2, \ldots, q^{\prime}\right)$;

(c) for every nonzero real eigenvalue $\alpha$ of $A+t(\mathrm{i} B)$ and for every index $\ell$ of $\alpha$, the following holds: if $w_{1}, \ldots, w_{k}$ are the distinct integers between $q^{\prime}+1$ and $q$ such that $\alpha_{w_{j}}=\alpha$ and $\ell_{w_{j}}=\ell, j=1,2, \ldots, k$, then there exists a permutation $\lambda$ of $\{1,2, \ldots, k\}$ with the property that the integers $\ell+\frac{1}{2}((1+$ $\left.\left.\kappa_{w_{\lambda(j)}}^{\prime \prime}\right)(\ell+1)+\eta_{w_{\lambda(j)}}^{\prime \prime}-\eta_{w_{j}}\right)$ are odd for $j=1,2, \ldots, k$.

(d) for each $j=1, \ldots, s$, either $\beta_{j}^{\prime \prime}=\beta_{j}$ or $\beta_{j}^{\prime \prime}=-\overline{\beta_{j}}$.

Then the complex hermitian-skewhermitian matrix pencils $A^{\prime}+t(-\mathrm{i}) B^{\prime}$ and $A^{\prime \prime}+$ $t(-\mathrm{i}) B^{\prime \prime}$ are $(\mathrm{V})_{\mathrm{i}}$-congruent.

The proof of Theorem 7.4 will be given separately in the next subsection.

Analogously to Corollary 6.4, we indicate a necessary and sufficient condition for the situation when (III)-congruence of complex hermitian pencils $A^{\prime}+t B^{\prime}$ and $A+t B$ is equivalent to the $(\mathrm{V})_{\mathrm{i}^{-}}$-congruence of the hermitian-skewhermitian pencils $A^{\prime}+t(-\mathrm{i}) B^{\prime}$ and $A+t(-\mathrm{i}) B$ :

COROLlary 7.5. The following two statements are equivalent for a complex hermitian pencil $A+t B, A=A^{*}, B=B^{*} \in \mathbb{C}^{n \times n}$ :

(1) For every complex hermitian pencil $A^{\prime}+t B^{\prime}$, the pencils $A^{\prime}+t B^{\prime}$ and $A+t B$ are (III)-congruent precisely when the hermitian-skewhermitian pencils $A^{\prime}+$ $t(-\mathrm{i}) B^{\prime}$ and $A+t(-\mathrm{i}) B$ are $(\mathrm{V})_{\mathrm{i}}$-congruent.

(2) All eigenvalues of $A+t B$ different from infinity are real, and in addition, in the complex Kronecker form of $A+t B$ there are no odd multiplicities corresponding to the eigenvalue at infinity and there are no even multiplicities corresponding to the eigenvalue zero.

The proof of Corollary 7.5 is obvious from Theorem 7.4.

As an application of Corollary 7.5 we present a result concerning comparison of the congruence over the complexes with the congruence over the quaternions, for complex matrices. 
TheOREM 7.6. Let $Y \in \mathbb{C}^{n \times n}$, and write $Y=Y_{0}+\mathrm{i} Y_{1}$, where $Y_{0}$ and $Y_{1}$ are complex hermitian matrices. The following statements are equivalent, where $\sigma$ is the nonstandard iaa such that $\sigma(\mathrm{i})=-\mathrm{i}$ :

(a) For any $X \in \mathbb{C}^{n \times}$, if $S^{\sigma} X S=Y$ holds for some invertible quaternionic matrix $S$, then $R^{*} X R=Y$ for some invertible complex matrix $R$;

(b) All complex eigenvalues of the hermitian matrix pencil $Y_{0}+t Y_{1}$ are real, and in addition, in the complex Kronecker form of $Y_{0}+t Y_{1}$ there are no odd multiplicities corresponding to the eigenvalue at infinity and there are no even multiplicities corresponding to the eigenvalue zero.

Proof. The proof follows by applying Corollary 7.5 to the matrix pencil $Y_{1}+t Y_{2}$, i.e., we take $A=Y_{1}, B=Y_{2}$. Let us provide some details. Assume (b) holds, and let $X \in \mathbb{C}^{n \times n}$ be such that $S^{\sigma} X S=Y$ for some invertible quaternionic matrix $S$. Write $X=X_{1}+\mathrm{i} X_{2}$, where $X_{1}$ and $X_{2}$ are complex hermitian. Note that every quaternionic matrix admits a unique decomposition as the sum of a $\sigma$-symmetric matrix and of a $\sigma$-skewsymmetric matrix. As a result, it follows from $S^{\sigma} X S=Y$ that

$$
S^{\sigma}\left(X_{1}+t(-\mathrm{i}) X_{2}\right) S=Y_{1}+t(-\mathrm{i}) Y_{2}, \quad \forall t \in \mathbb{R},
$$

in other words, the matrix pencils $X_{1}+t(-\mathrm{i}) X_{2}$ and $Y_{1}+t(-\mathrm{i}) Y_{2}$ are $(\mathrm{V})_{\mathrm{i}}$-congruent. By Corollary 7.5, there exists an invertible complex matrix $R$ such that

$$
R^{*}\left(X_{1}+t X_{2}\right) R=Y_{1}+t Y_{2}, \quad \forall t \in \mathbb{R} .
$$

But then equality (7.6) holds also for all $t \in \mathbb{C}$, in particular, it holds for $t=\mathrm{i}$, and the statement (a) follows.

7.3. Proof of Theorem 7.4. The proof will be patterned on that of Theorem 6.3. We start with an analogue of Lemma 6.5.

Lemma 7.7. In the statements $(\mathrm{C})-(\mathrm{G})$ below, $\eta, \eta^{\prime}, \kappa^{\prime}$ are signs \pm 1 and $\alpha$ is a nonzero real number. We have:

(A) $F_{k}+t(-\mathrm{i}) G_{k}$ is $(\mathrm{V})_{\mathrm{i}}$-congruent to $-F_{k}+t \mathrm{i} G_{k}$ for odd $k$;

(A') $F_{k}+t(-\mathrm{i}) G_{k}$ is not $(\mathrm{V})_{\mathrm{i}}$-congruent to $-F_{k}+$ ti $G_{k}$ for even $k$;

(B) $t(-\mathrm{i}) F_{\ell}+G_{\ell}$ is $(\mathrm{V})_{\mathrm{i}}$-congruent to $\mathrm{ti} F_{\ell}-G_{\ell}$ for even $\ell$;

(B') $t(-\mathrm{i}) F_{\ell}+G_{\ell}$ is not $(\mathrm{V})_{\mathrm{i}}$-congruent to $\mathrm{ti} F_{\ell}-G_{\ell}$ for odd $\ell$;

(C) For $\eta^{\prime} \neq \eta$ and $\kappa^{\prime}=1$, the pencil

$$
\eta^{\prime}\left(\left(-i t+\kappa^{\prime} \alpha\right) F_{\ell}+G_{\ell}\right)
$$

is not $(\mathrm{V})_{\mathrm{i}}$-congruent to

$$
\eta\left((-\mathrm{i} t+\alpha) F_{\ell}+G_{\ell}\right)
$$

(D) For $\kappa^{\prime}=-1$, $\ell$ even, and $\eta^{\prime}=-\eta$, the pencil (7.7) is $(\mathrm{V})_{\mathrm{i}}$-congruent to (7.8);

(E) For $\kappa^{\prime}=-1, \ell$ even, and $\eta^{\prime}=\eta$, the pencil $(7.7)$ is not $(\mathrm{V})_{\mathrm{i}}$-congruent to $(7.8)$

(F) For $\kappa^{\prime}=-1, \ell$ odd and $\eta^{\prime}=\eta$, the pencil $(7.7)$ is $(\mathrm{V})_{\mathrm{i}}$-congruent to (7.8);

(G) For $\kappa^{\prime}=-1, \ell$ odd and $\eta^{\prime}=-\eta,(7.7)$ is not $(\mathrm{V})_{\mathrm{i}}$-congruent to $(7.8)$; 
(H) If $\beta \in \mathbb{C} \backslash \mathbb{R}$, then the matrix pencil

$$
\left[\begin{array}{cc}
0 & (-\mathrm{i} t+\beta) F_{m}+G_{m} \\
(-\mathrm{i} t+\bar{\beta}) F_{m}+G_{m} & 0
\end{array}\right]
$$

is $(\mathrm{V})_{\mathrm{i}}$-congruent to

$$
\left[\begin{array}{cc}
0 & (-\mathrm{i} t-\bar{\beta}) F_{m}+G_{m} \\
(-\mathrm{i} t-\beta) F_{m}+G_{m} & 0
\end{array}\right]
$$

Proof. Parts (A), ( $\left.\mathrm{A}^{\prime}\right),(\mathrm{B}),\left(\mathrm{B}^{\prime}\right),(\mathrm{C})$ follow from [17, Theorem 3.1]; note that the $\mathbb{H}$-Kronecker form of (7.7), as well as that of (7.8), consists of one Jordan block with eigenvalue i $\alpha$ (up to similarity). For the part (D) observe the equality

$$
\operatorname{diag}(-\mathrm{j}, \mathrm{j}, \ldots,-\mathrm{j}, \mathrm{j})\left((-\mathrm{i} t-\alpha) F_{\ell}+G_{\ell}\right) \operatorname{diag}(-\mathrm{j}, \mathrm{j}, \ldots,-\mathrm{j}, \mathrm{j})=-\left((-\mathrm{i} t+\alpha) F_{\ell}+G_{\ell}\right) .
$$

Consider (E). By the already proved part (D), the matrix pencils

$$
\eta^{\prime}\left((-\mathrm{i} t-\alpha) F_{\ell}+G_{\ell}\right) \quad \text { and } \quad-\eta^{\prime}\left((-\mathrm{i} t+\alpha) F_{\ell}+G_{\ell}\right)
$$

are $(\mathrm{V})_{\mathrm{i}}$-congruent; but

$$
-\eta^{\prime}\left((-\mathrm{i} t+\alpha) F_{\ell}+G_{\ell}\right)=-\eta\left((-\mathrm{i} t+\alpha) F_{\ell}+G_{\ell}\right),
$$

which is not $(\mathrm{V})_{\mathrm{i}}$-congruent to $(7.8)$ by the same [17, Theorem 3.1]. Statement $(\mathrm{F})$ (where we take $\eta=\eta^{\prime}=-1$ without loss of generality) is proved by the equality

$\operatorname{diag}(\mathrm{j},-\mathrm{j}, \mathrm{j}, \ldots,-\mathrm{j}, \mathrm{j})\left((\mathrm{it}+\alpha) F_{\ell}-G_{\ell}\right) \operatorname{diag}(\mathrm{j},-\mathrm{j}, \mathrm{j}, \ldots,-\mathrm{j}, \mathrm{j})=-\left((-\mathrm{i} t+\alpha) F_{\ell}+G_{\ell}\right)$, where $\ell$ is odd. Next, consider (G), taking $\eta=1, \eta^{\prime}=-1$. Then

$$
\eta^{\prime}\left(\left(-\mathrm{i} t+\kappa^{\prime} \alpha\right) F_{\ell}+G_{\ell}\right)=(\mathrm{i} t+\alpha) F_{\ell}-G_{\ell}
$$

is $(\mathrm{V})_{\mathrm{i}}$-congruent to the negative of (7.8), in view of the already proved part $(\mathrm{F})$. But (7.8) is not $(\mathrm{V})_{\mathrm{i}}$-congruent to its negative by [17, Theorem 3.1], as the block (7.8) is $\mathbb{H}$-strictly equivalent to a Jordan block with eigenvalue $\alpha \mathrm{i}$ (up to similarity).

It remains to prove $(\mathrm{H})$. Let $X \in \mathbb{H}^{m \times m}$ be an invertible matrix such that

$$
X^{-1}(\bar{i} \bar{\beta} I+\mathrm{i} F G) X=-\mathrm{i} \beta I+\mathrm{i} F G,
$$

where we set $F=F_{m}$ and $G=G_{m}$ for short, and define $Y \in \mathbb{H}^{m \times m}$ by the equality

$$
Y^{\sigma}=-\mathrm{i} F X^{-1} \mathbf{i} F .
$$

Then the following equation is easily verified:

$$
\left[\begin{array}{cc}
X^{\sigma} & 0 \\
0 & Y^{\sigma}
\end{array}\right]\left[\begin{array}{cc}
0 & (-\mathrm{i} t+\beta) F+G \\
(-\mathrm{i} t+\bar{\beta}) F+G & 0
\end{array}\right]\left[\begin{array}{cc}
X & 0 \\
0 & Y
\end{array}\right]
$$




$$
=\left[\begin{array}{cc}
0 & (-\mathrm{i} t-\bar{\beta}) F+G \\
(-\mathrm{i} t-\beta) F+G & 0
\end{array}\right],
$$

which proves $(\mathrm{H})$.

Summarizing the parts $(\mathrm{C})$ - $(\mathrm{G})$ of Lemma 7.7, we obtain:

Corollary 7.8. For signs $\eta, \eta^{\prime}, \kappa^{\prime} \in\{1,-1\}$, positive integer $\ell$, and $\alpha \in \mathbb{R} \backslash\{0\}$, the matrix pencils $(7.7)$ and $(7.8)$ are $(\mathrm{V})_{\mathrm{i}}$-congruent if and only if the integer

$$
\ell+\frac{1}{2}\left(\left(1+\kappa^{\prime}\right)(\ell+1)+\eta^{\prime}-\eta\right)
$$

is odd.

Proof of Theorem 7.4. The direct statement: As in the proof of Theorem 6.3, we see that the canonical form of the complex hermitian matrix pencil $A^{\prime}+t\left(\mathrm{i} B^{\prime}\right)$ under (III)-congruence must have the structure as in (6.6). Now the proof proceeds analogously to the proof of Theorem 6.3, using Lemma 7.7 and the canonical form for symmetric-skewsymmetric quaternionic pencils under $(\mathrm{V})_{\mathrm{i}}{ }^{-}$-congruence (see $[17$, Theorem 3.1]) instead of Theorem 5.3 in [18].

The converse statement follows easily from Lemma 7.7 and Corollary 7.8.

\section{REFERENCES}

[1] N. Bourbaki. Éléments de mathématique. Livre II: Algèbre. Chapitre 9: Formes sesquilinéaires et formes quadratiques, Hermann, Paris, 1959.

[2] E. Brieskorn. Lineare Algebra und analytische Geometrie, Volumes I and II. Friedr. Vieweg and Sohn, Braunschweig, 1985.

[3] D. Z. Djokovic. Classification of pairs consisting of a linear and a semilinear map. Linear Multilinear Algebra, 29:147-165, 1978.

[4] D. Z. Djokovic, J. Patera, P. Winternitz, and H. Zassenhaus. Normal forms of elements of classical real and complex Lie and Jordan algebras. J. Math. Phys., 24:1363-1374, 1983.

[5] D. R. Farenick and B. A. F. Pidkowich. The spectral theorem in quaternions. Linear Algebra Appl., 371:75-102, 2003.

[6] F. R. Gantmacher. The Theory of Matrices, Vols. 1 and 2, Chelsea, New York, 1959. (Translation from Russian.)

[7] F. R. Gantmacher. Applications of the Theory of Matrices, Interscience Publishers, New York, 1959. (Translation of part II of the Russian original.)

[8] I. Gohberg, P. Lancaster, and L. Rodman. Invariant Subspaces of Matrices with Applications. John Wiley, 1986; republication, SIAM, 2006.

[9] R. A. Horn and V. V. Sergeichuk. Canonical forms for complex matrix congruence and *congruence. Linear Algebra Appl., 416:1010-1032, 2006.

[10] M. Karow. Self-adjoint operators and pairs of Hermitian forms over the quaternions. Linear Algebra Appl., 299:101-117, 1999.

[11] P. Lancaster and L. Rodman. Canonical forms for hermitian matrix pairs under strict equivalence and congruence. SIAM Review, 47:407-443, 2005.

[12] P. Lancaster and L. Rodman. Canonical forms for symmetric/skew-symmetric real matrix pairs under strict equivalence and congruence. Linear Algebra Appl., 406:1-76, 2005.

[13] R. Pereira, P. Rocha, and P. Vettori. Algebraic tools for the study of quaternionic behavioral systems. Linear Algebra Appl., 400:121-140, 2005.

[14] R. Pereira and P. Vettori. Stability of quaternionic linear systems. IEEE Trans. Automat. Control, 51:518-523, 2006.

[15] R. von Randow. The involutory antiautomorphisms of the quaternion algebra. American Mathematical Monthly, 74:699-700, 1967. 
[16] L. Rodman. Canonical forms for symmetric and skew-symmetric quaternionic matrix pencils. Operator Theory: Advances and Applications, 176:199-254, 2007.

[17] L. Rodman. Canonical forms for mixed symmetric-skewsymmetric quaternion matrix pencils. To appear in Linear Algebra Appl.

[18] L. Rodman. Pairs of hermitian and skew-hermitian quaternionic matrices: canonical forms and their applications. To appear in Linear Algebra Appl.

[19] V. V. Sergeichuk. Classification problems for systems of forms and linear mappings. Math. USSR-Izv., 31:481-501, 1988. (Translation from Russian.)

[20] V. V. Sergeichuk. Classification of sesquilinear forms, pairs of Hermitian forms, self-conjugate and isometric operators over the division ring of quaternions. Math. Notes, 49:409-414, 1991. (Translation from Russian.)

[21] R. C. Thompson. Pencils of complex and real symmetric and skew matrices. Linear Algebra Appl., 147:323-371, 1991.

[22] Z.-X. Wan. Geometry of Matrices. World Scientific Publishing, River Edge, NJ, 1996.

[23] F. Zhang. Quaternions and matrices of quaternions. Linear Algebra Appl., 251:21-57, 1997. 